\title{
SIMULTANEOUS NONVANISHING OF DIRICHLET $L$-FUNCTIONS AND TWISTS OF HECKE-MAASS $L$-FUNCTIONS IN THE CRITICAL STRIP
}

\author{
Keiju Sono \\ Ehime University \\ Dogo-Himata, Matsuyama, Ehime, Japan; sono.keiju.jk@ehime-u.ac.jp
}

\begin{abstract}
In this paper, we consider the moment of the products of primitive Dirichlet $L$ functions and $L$-functions associated with a Hecke-Maass form of $\operatorname{SL}(2, \mathbf{Z})$ twisted by primitive Dirichlet characters. We prove that for any Hecke-Maass form $f$ of $\operatorname{SL}(2, \mathbf{Z})$ and $s_{0}=\sigma_{0}+i t_{0}$ with $1 / 2 \leq \sigma_{0}<1, L\left(s_{0}, f \otimes \chi\right) L\left(s_{0}, \chi\right) \neq 0$ holds for some primitive Dirichlet character $\chi$ if the conductor of $\chi$ is prime and sufficiently large. In particular, we show that unconditionally $L(1 / 2+i t, f \otimes \chi) L(1 / 2+i t, \chi) \neq 0$ for some primitive Dirichlet character modulo $q$ for prime values of $q$ satisfying $q \gg(1+|t|)^{255+\epsilon}$. If we assume the Ramanujan-Petersson conjecture, the same statement is valid for any prime values of $q$ such that $q \gg(1+|t|)^{15+\epsilon}$.
\end{abstract}

\section{Introduction}

It is an important problem to determine whether an automorphic $L$-function is vanishing at the central point of its functional equation. This problem is related to several deep problems in number theory, including the Birch and Swinnerton-Dyer conjecture, the nonvanishing of theta lifting, and the nonexistence of Landau-Siegel zeros. There has been a number of results in this area. For example, Iwaniec and Sarnak [10] proved that at least one third of Dirichlet $L$-functions do not vanish at $s=1 / 2$. In another paper [11] the same authors further showed that at least half of the values of $L(1 / 2, f)$, with $f$ varying among the even holomorphic newforms of a fixed even integral weight for $\Gamma_{0}(N)$, are positive as $N \rightarrow \infty$. Soundararajan [21] proved that at least seven-eighths of primitive quadratic $L$-functions do not vanish at $s=1 / 2$. The problem of simultaneous nonvanishing of $L$-functions is also important. Briefly, this problem concerns whether $L\left(s_{0}, f\right) L\left(s_{0}, g\right) \neq 0$ holds or not for some fixed point $s_{0}$, where $L(s, f)$ and $L(s, g)$ are two $L$-functions that are related to each other. This problem has been extensively investigated (see, for example, $[1,3,4,7,12,14,15,16,18,17,19,22,23])$. Some of the results that are particularly related to the context of this paper are described as follows.

Liu [16] considered the simultaneous nonvanishing of Dirichlet $L$-functions and twists of automorphic $L$-functions. Let $\mathcal{P}$ be the set of prime numbers and for $Q \geq 2$,

$$
\mathcal{D}:=\left\{q \mid q=p r, Q^{\frac{3}{4}}<p \leq 2 Q^{\frac{3}{4}}, Q^{\frac{1}{4}}<r \leq 2 Q^{\frac{1}{4}}, p, r \in \mathcal{P}\right\} .
$$

Let $\pi$ be a unitary cuspidal automorphic representation of $\mathrm{GL}_{2}\left(\mathbf{A}_{\mathbf{Q}}\right)$. Then Liu's result asserts that for any $\epsilon>0$, asymptotically

$$
\sum_{q \in \mathcal{D}} \sum_{\chi(\bmod q)}^{+} L\left(\frac{1}{2}, \pi \otimes \chi\right) L\left(\frac{1}{2}, \chi\right)=A(Q)+O\left(Q^{\frac{15}{8}+\epsilon}\right),
$$

https://doi.org/10.5186/aasfm.2019.4464

2010 Mathematics Subject Classification: Primary 11M06.

Key words: Simultaneous nonvanishing, Hecke-Maass form. 
where $\sum^{+}$denotes the summation over even primitive Dirichlet characters and $A(Q):=(1 / 2) \sum_{q \in \mathcal{D}} q \asymp Q^{2} / \log ^{2} Q$. Consequently for each sufficiently large value of $Q$, there exists a primitive Dirichlet character $\chi(\bmod q)$ with $Q<q \leq 4 Q$ such that $L(1 / 2, \pi \otimes \chi) L(1 / 2, \chi) \neq 0$.

Inspired by Liu's work, Das and Khan [4] considered the simultaneous nonvanishing of Dirichlet $L$-functions and twists of Hecke-Maass $L$-functions of $\operatorname{SL}(2, \mathbf{Z})$. They proved that for a Hecke-Maass form $f$ for $\mathrm{SL}(2, \mathbf{Z})$ and prime values of $q$, one has

$$
\sum_{\chi(\bmod q)}^{+} L\left(\frac{1}{2}, f \otimes \chi\right) \overline{L\left(\frac{1}{2}, \chi\right)}=\frac{q-2}{2} L(1, f)+O\left(q^{\frac{7}{8}+\frac{7}{64}+\epsilon}\right),
$$

where $\epsilon>0$ is arbitrarily small. The number $7 / 64$ in the exponent is a consequence of the Kim-Sarnak bound (see the appendix 2 of [13]) towards the RamanujanPetersson conjecture. Since $L(1, f) \neq 0$ (see [9], Lemma 5.9), their result implies that for every sufficiently large values of $q$, there exists an even primitive character $\chi(\bmod q)$ such that $L(1 / 2, f \otimes \chi) L(1 / 2, \chi) \neq 0$. In some respects, their results are stronger than the result presented by Liu, because the conductor of the characters is fixed in this case. Recently Sun [22] considered the case in which the conductor $q$ is a product of two primes. On the other hand, Blomer, Fouvry, Kowalski, Michel and Milićević [3] obtained

$$
\sum_{\chi(\bmod q)}^{*} L\left(\frac{1}{2}, f \otimes \chi\right) \overline{L\left(\frac{1}{2}, \chi\right)}^{2}=\frac{(q-2) L(1, f)^{2}}{\zeta(2)}+O\left(q^{-\delta}\right)
$$

for some $\delta>0$ in the same setting as above. The notation $\sum^{*}$ denotes the summation over all primitive characters. The same conclusion regarding the simultaneous nonvanishing can be deduced from this asymptotic formula.

There are several results on nonvanishing of automorphic $L$-functions at a general point. Let $F$ be a number field, $S$ be a finite set of places of $F$, and $\pi$ be a unitary cuspidal automorphic representation of $\operatorname{GL}(n)$. In the cases of $n=1$ and 2, Rohrlich [20] proved that there are infinitely many primitive ray class characters $\chi$ of $F$ such that $L\left(s_{0}, \pi \otimes \chi\right) \neq 0$, where $s_{0}$ is a general point in the complex plane. Barthel and Ramakrishnan [2] proved the same results for $n \geq 3$ under the assumptions that $\pi$ is tempered (i.e. satisfies the Ramanujan conjecture) and $\Re\left(s_{0}\right)>1-2 /(n+1)$. Akbary [1] studied the simultaneous nonvanishing of Dirichlet twists of newforms at a general point. He proved that for newforms $f \in \mathcal{S}_{k}\left(\Gamma_{0}(M), \psi\right), g \in \mathcal{S}_{k}\left(\Gamma_{0}(N), \eta\right)$ and a complex number $s_{0}=\sigma_{0}+i t_{0}$ with $\sigma_{0}>1 / 2$,

$$
\begin{aligned}
& \sum_{\substack{q \leq Q, q: \operatorname{prime} \\
(q, M N)=1}} \sum_{(\bmod q)}^{*} L\left(s_{0}, f \otimes \chi\right) \overline{L\left(s_{0}, g \otimes \chi\right)} \\
& =\frac{Q^{2}}{2 \log Q} \frac{\varphi(M N)}{M N} L\left(2 \sigma_{0}, f \otimes g\right)+O\left(\frac{Q^{2}}{(\log Q)^{2}}\right)
\end{aligned}
$$

as $Q \rightarrow \infty$. This indicates that for any $s_{0}=\sigma_{0}+i t_{0}$ with $1 / 2<\sigma_{0} \leq 1$, there exists a primitive Dirichlet character $\chi$ for which $L\left(s_{0}, f \otimes \chi\right) L\left(s_{0}, g \otimes \chi\right) \neq 0$. This theorem is related to the case of $n=4$ of the result of Barthel and Ramakrishnan above, and this surpasses the bound $\sigma_{0}>1-2 /(4+1)$. However, it has not been proved that a similar result is valid on the line $\sigma_{0}=1 / 2$. It is mentioned in [1] that such a result needs a more elaborate treatment of the error terms in Proposition 2.5 of [1]. 
The objective of this paper is to extend the results of Das and Khan [4] as described above. We provide an asymptotic formula for the mean value of products of Dirichlet $L$-functions and twists of a Hecke-Maass $L$-function of $\operatorname{SL}(2, \mathbf{Z})$ at a general point $s_{0}=\sigma_{0}+i t_{0}$ with $1 / 2 \leq \sigma_{0}<1$. The main theorem of this paper is stated as follows.

Theorem 1.1. Let $f$ be a Hecke-Maass form of $S L(2, \mathbf{Z})$ and $1 / 2 \leq A_{0}<1$ be an arbitrary fixed number. For any prime values of $q$ and $s_{0}=\sigma_{0}+i t_{0}$ with $1 / 2 \leq \sigma_{0} \leq A_{0}<1$, we have

$$
\sum_{\chi(\bmod q)}^{+} L\left(s_{0}, f \otimes \chi\right) \overline{L\left(s_{0}, \chi\right)}=\frac{1}{2} L\left(2 \sigma_{0}, f\right) q+O\left(\sum_{i=1}^{7} E_{i}\right)
$$

where $\sum^{+}$denotes the summation over even primitive characters, and $L(s, f)$ denotes the $L$-function associated to $f$. The error terms $E_{1}, \cdots, E_{7}$ are given by

$$
\begin{aligned}
& E_{1}=q^{1-\theta-2 \sigma_{0}+\epsilon}(q \tau)^{\theta+\epsilon}, \quad E_{2}=q^{-\frac{5}{4}+\frac{3}{2} \sigma_{0}+\epsilon}(q \tau)^{\frac{3}{2}-\frac{3}{2} \sigma_{0}}, \quad E_{3}=(q \tau)^{1-\frac{\sigma_{0}}{2}+\theta+\epsilon}, \\
& E_{4}=q^{-\frac{1}{2}}(q \tau)^{\frac{3}{2}-\frac{\sigma_{0}}{2}+\epsilon}, \quad E_{5}=q^{\frac{1}{2}}(q \tau)^{\frac{5}{4}+\frac{15}{26} \theta-\frac{87}{52} \sigma_{0}+\epsilon}, \quad E_{6}=q^{-1}(q \tau)^{\frac{5}{2}-\frac{5}{4} \sigma_{0}+\epsilon}, \\
& E_{7}=q^{-1}(q \tau)^{-\frac{87}{52} \sigma_{0}+\frac{37}{26} \theta+\frac{139}{52}+\epsilon} .
\end{aligned}
$$

Here, $\epsilon>0$ is arbitrarily small, $\tau:=\left|t_{0}\right|+3$ and $\theta$ in the exponents represent the best bound towards the Ramanujan-Petersson conjecture for $f$, and can currently be taken as $\theta=7 / 64$. The implied constant may depend on both $f$ and $A_{0}$, but is independent of $q$ and $\tau$.

It should be mentioned that our Theorem 1.1 does not recover (1.1) completely. Indeed, by putting $\sigma_{0}=1 / 2, \tau=3$ and $\theta=7 / 64$, it follows that the error term in Theorem 1.1 becomes $O\left(q^{\frac{127}{128}+\epsilon}\right)$ (the $E_{7}$ takes the maximal value). On the other hand, the error term in the asymptotic formula (1.1) is $O\left(q^{\frac{126}{128}+\epsilon}\right)$. The reason of this subtle difference is that the author used slightly different values of $N$ and $M$ to evaluate the contribution of the terms involving $S_{2} S_{3}$ (see the proof of Lemma 3.5). The argument of Case II in [4] seems to be the most complicated part of that paper, and our choice of these values enables us to treat these terms in a more direct way.

Let $M\left(\sigma_{0}, \theta\right)$ be the maximum of $\theta /\left(2 \sigma_{0}\right), 2\left(1-\sigma_{0}\right),\left(2-\sigma_{0}+2 \theta\right) /\left(\sigma_{0}-2 \theta\right)$, $\left(3-\sigma_{0}\right) / \sigma_{0},\left(65+30 \theta-87 \sigma_{0}\right) /\left(87 \sigma_{0}-30 \theta-39\right),\left(10-5 \sigma_{0}\right) /\left(5 \sigma_{0}-2\right)$ and $\left(-87 \sigma_{0}+\right.$ $74 \theta+139) /\left(87 \sigma_{0}-74 \theta-35\right)$. It can be easily confirmed that the main term in $(1.2)$ dominates the error terms if $q \gg_{f, A_{0}} \tau^{M\left(\sigma_{0}, \theta\right)+\epsilon}$. Moreover, we have $M(1 / 2,7 / 64)=$ $255, M(1 / 2,0)=15$. Hence we obtain the following corollary:

Corollary 1.2. For a Hecke-Maass form $f$ of $S L(2, \mathbf{Z})$, there exists a primitive Dirichlet character $\chi$ modulo $q$ for which $L\left(s_{0}, f \otimes \chi\right)$ and $L\left(s_{0}, \chi\right)$ do not vanish if the prime $q$ satisfies $q \gg_{f, A_{0}} \tau^{M\left(\sigma_{0}, \theta\right)+\epsilon}$. In particular, for any $t \in \mathbf{R}$, there exists a primitive Dirichlet character $\chi$ modulo $q$ for which $L(1 / 2+i t, f \otimes \chi)$ and $L(1 / 2+i t, \chi)$ do not vanish if the prime $q$ satisfies $q \gg_{f}(1+|t|)^{255+\epsilon}$. Further, if we assume the Ramanujan-Petersson conjecture $(\theta=0)$, the same statement is valid if the prime $q$ satisfies $q \gg_{f}(1+|t|)^{15+\epsilon}$.

Using Hölder's inequality, we obtain a lower bound for the proportion of the characters for which the $L$-functions are nonvanishing at $s=s_{0}$. 
Theorem 1.3. Let $f$ be a Hecke-Maass form of $S L(2, \mathbf{Z})$ and $q$ be a prime. For $s_{0}=\sigma_{0}+i t_{0}$ with $1 / 2 \leq \sigma_{0}<1$, put $\tau=\left|t_{0}\right|+3$,

$$
\mathcal{A}_{f, s_{0}}=\left\{\chi(\bmod q) \mid L\left(s_{0}, f \otimes \chi\right) L\left(s_{0}, \chi\right) \neq 0, \chi(-1)=1, \chi: \text { primitive }\right\} .
$$

Then, for $q \gg \tau^{M\left(\sigma_{0}, \theta\right)+\epsilon}$, we have

$$
\left|\mathcal{A}_{f, s_{0}}\right| \gg \begin{cases}\left(q \tau^{-3}\right)^{1-\epsilon} & \left(\sigma_{0}=\frac{1}{2}\right), \\ q \tau^{-3} & \left(\frac{1}{2}<\sigma_{0}<1\right)\end{cases}
$$

for any $\epsilon>0$. The implied constants may be dependent on $f, \epsilon$ and $\sigma_{0}$, but they are independent of $q$ and $\tau$.

In particular, for any fixed $s_{0}=\sigma_{0}+i t_{0}$ with $1 / 2<\sigma_{0}<1$, there exists a positive proportion of even primitive Dirichlet characters $\chi(\bmod q)$ for which $L\left(s_{0}, f \otimes \chi\right) L\left(s_{0}, \chi\right) \neq 0$. This kind of result is not found in the paper of Das and Khan [4]. The author is sure that the same statement will be valid for $\sigma_{0}=1 / 2$, but the proof will require several elaborate arguments on mollified moments of $L$ functions at $s_{0}=1 / 2+i t_{0}$.

\section{Preliminaries}

Here, we prove Theorem 1.1 for even Hecke-Maass forms, as the proof for odd forms is entirely similar. Let $\chi$ be an even primitive Dirichlet character modulo $q$. The Dirichlet $L$-function associated to $\chi$ is defined by

$$
L(s, \chi)=\sum_{n=1}^{\infty} \frac{\chi(n)}{n^{s}}
$$

for $\Re(s)>1$. This function is continued holomorphically to the whole $s$-plane and satisfies the functional equation

$$
\Lambda(s, \chi)=\frac{\tau(\chi)}{\sqrt{q}} \Lambda(1-s, \bar{\chi})
$$

where

$$
\Lambda(s, \chi)=\left(\frac{q}{\pi}\right)^{\frac{s}{2}} \Gamma\left(\frac{s}{2}\right) L(s, \chi)
$$

and $\tau(\chi)=\sum_{a(\bmod q)}^{*} \chi(a) \exp (2 \pi i a / q)$ is the Gauss sum.

Next, let $f$ be an even Hecke-Maass form for the full modular group $\operatorname{SL}(2, \mathbf{Z})$. The eigenvalue of Laplacian is expressed by $1 / 4+T_{f}^{2}$, where $T_{f}$ is a real number. We denote the $n$-th Hecke eigenvalue of $f$ by $\lambda_{f}(n)$. For the Dirichlet character $\chi$ above, the Dirichlet twist of Hecke-Maass $L$-function is defined by

$$
L(s, f \otimes \chi)=\sum_{n=1}^{\infty} \frac{\lambda_{f}(n) \chi(n)}{n^{s}}
$$

for $\Re(s)>1$. This function is continued holomorphically to the whole $s$-plane and satisfies the functional equation

$$
\Lambda(s, f \otimes \chi)=\frac{\tau(\chi)^{2}}{q} \Lambda(1-s, f \otimes \bar{\chi})
$$

where

$$
\Lambda(s, f \otimes \chi)=\left(\frac{q}{\pi}\right)^{s} \Gamma\left(\frac{s+i T_{f}}{2}\right) \Gamma\left(\frac{s-i T_{f}}{2}\right) L(s, f \otimes \chi)
$$


Let us prepare several lemmas on Dirichlet characters and Fourier coefficients of Hecke-Maass forms.

2.1. Orthogonality of Dirichlet characters. To compute the summation averaged over primitive Dirichlet characters, we use the following property of characters.

Lemma 2.1. Let $q$ be a prime number and $n, m$ be positive integers such that $(n m, q)=1$. Then,

$$
\sum_{\chi(\bmod q)}^{*} \chi(n) \bar{\chi}(m)= \begin{cases}q-2 & (n \equiv m(\bmod q)) \\ -1 & (\text { otherwise })\end{cases}
$$
[5].

The proof of this statement can be found in many textbooks. For example, see

2.2. Several properties of Fourier coefficients. We frequently use the following individual bound, proved by Kim and Sarnak [13]:

Lemma 2.2. For any $\epsilon>0$, we have

$$
\left|\lambda_{f}(n)\right| \ll_{\epsilon} n^{\theta+\epsilon}
$$

where $\theta=7 / 64$.

To evaluate sums involving $\lambda_{f}(n)$, we almost always adapt Lemma 2.2 above. It should be mentioned that several estimates might be improved if we instead combine Lemma 2.3 below on the average of $\left|\lambda_{f}(n)\right|$ with partial summation:

It follows from the Rankin-Selberg theory that the Fourier coefficients satisfy

$$
\sum_{n \leq x}\left|\lambda_{f}(n)\right|^{2} \ll x
$$

Combining this with the Cauchy-Schwarz inequality, we obtain the following upper bound.

Lemma 2.3. We have

$$
\sum_{n \leq x}\left|\lambda_{f}(n)\right| \ll x
$$

as $x \rightarrow \infty$.

Next, we prepare the following Voronoi summation formula for Hecke-Maass form of $\mathrm{SL}(2, \mathbf{Z})$, proved by Godber [6, Theorem 3.2]:

Lemma 2.4. Let $q$ be a positive integer and $\psi$ be a smooth function compactly supported in $\mathbf{R}_{>0}$. Let $d$ and $\bar{d}$ be integers satisfying $(q, d)=1, d \bar{d} \equiv 1(\bmod q)$. Then, we have

$$
\begin{aligned}
& \sum_{n=1}^{\infty} \lambda_{f}(n) e\left(\frac{n \bar{d}}{q}\right) \psi\left(\frac{n}{N}\right) \\
& =q \sum_{n=1}^{\infty} \frac{\lambda_{f}(n)}{n} e\left(\frac{n d}{q}\right) \Psi_{+}\left(\frac{n N}{q^{2}}\right)+q \sum_{n=1}^{\infty} \frac{\lambda_{f}(n)}{n} e\left(-\frac{n d}{q}\right) \Psi_{-}\left(\frac{n N}{q^{2}}\right)
\end{aligned}
$$

where $\Psi_{ \pm}$are defined by

$$
\Psi_{ \pm}(s)=\frac{1}{2 \pi i} \int_{(\sigma)}\left(\pi^{2} x\right)^{-s} G_{ \pm}(s) \tilde{\psi}(-s) d s
$$


for $\sigma>-1$, respectively. Here, $\tilde{\psi}$ denotes the Mellin transform of $\psi$ and $G_{ \pm}(s)$ are defined by

$$
2 \pi G_{ \pm}(s)=\frac{\Gamma\left(\frac{1+s+i T_{f}}{2}\right) \Gamma\left(\frac{1+s-i T_{f}}{2}\right)}{\Gamma\left(\frac{-s+i T_{f}}{2}\right) \Gamma\left(\frac{-s-i T_{f}}{2}\right)} \pm \frac{\Gamma\left(\frac{1+s+i T_{f}+1}{2}\right) \Gamma\left(\frac{1+s-i T_{f}+1}{2}\right)}{\Gamma\left(\frac{-s+i T_{f}+1}{2}\right) \Gamma\left(\frac{-s-i T_{f}+1}{2}\right)},
$$

respectively.

Next, we use the following result [8, Theorem 8.1], which indicates that the Fourier coefficients of $f$ are orthogonal to additive characters on average:

Lemma 2.5. For any real number $\alpha$, we have

$$
\sum_{n \leq N} \lambda_{f}(n) e(\alpha n) \ll_{\epsilon} N^{\frac{1}{2}+\epsilon} .
$$

Here, the implied constant is independent of $\alpha$.

2.3. Approximate functional equations. To compute the moment in Theorem 1.1, we need some approximate functional equations for $L(s, \chi)$ and $L(s, f \otimes \chi)$. The following two lemmas are obtained by applying Theorem 5.3 and Proposition 5.4 of the textbook of Iwaniec and Kowalski [9] to these $L$-functions. Hereafter we put $\tau:=\left|t_{0}\right|+3$.

Lemma 2.6. For $s_{0}=\sigma_{0}+i t_{0}$ with $1 / 2 \leq \sigma_{0}<1$, we have

$$
\begin{aligned}
& L\left(s_{0}, \chi\right) \\
& =\sum_{n} \frac{\chi(n)}{n^{s_{0}}} V_{s_{0}}\left(\frac{n}{\sqrt{q}}\right)+\tau(\chi) q^{-s_{0}} \frac{\gamma\left(1-s_{0}\right)}{\gamma\left(s_{0}\right)} \sum_{n} \frac{\bar{\chi}(n)}{n^{1-s_{0}}} V_{1-s_{0}}\left(\frac{n}{\sqrt{q}}\right) .
\end{aligned}
$$

Here, $\gamma(s):=\pi^{-\frac{s}{2}} \Gamma(s / 2)$ and

$$
V_{s_{0}}(x)=\frac{1}{2 \pi i} \int_{(3)}(\sqrt{\pi} x)^{-u} \frac{\Gamma\left(\frac{s_{0}+u}{2}\right)}{\Gamma\left(\frac{s_{0}}{2}\right)} e^{u^{2}} \frac{d u}{u} .
$$

The function $V_{s_{0}}(x)$ and its derivatives are bounded by

$$
\begin{aligned}
V_{s_{0}}(x) & \ll_{A} \min \left\{1,(x / \sqrt{\tau})^{-A}\right\}, \\
V_{s_{0}}^{(l)}(x) & \ll x^{-l} .
\end{aligned}
$$

The implied constants depend only on $\sigma_{0}, A$ and $l$.

Lemma 2.7. Let $f$ be an even Hecke-Maass form for $S L(2, \mathbf{Z})$ and $\chi$ be an even primitive character modulo $q$. Then, for $s_{0}=\sigma_{0}+i t_{0}$ with $1 / 2 \leq \sigma_{0}<1$, we have

$$
\begin{aligned}
L\left(s_{0}, f \otimes \chi\right)= & \sum_{n} \frac{\lambda_{f}(n) \chi(n)}{n^{s_{0}}} W_{s_{0}}\left(\frac{n}{q}\right) \\
& +\tau(\chi)^{2} q^{-2 s_{0}} \frac{\tilde{\gamma}\left(1-s_{0}\right)}{\tilde{\gamma}\left(s_{0}\right)} \sum_{n} \frac{\lambda_{f}(n) \bar{\chi}(n)}{n^{1-s_{0}}} W_{1-s_{0}}\left(\frac{n}{q}\right) .
\end{aligned}
$$

Here, $\tilde{\gamma}(s):=\pi^{-s} \Gamma\left(\left(s+i T_{f}\right) / 2\right) \Gamma\left(\left(s-i T_{f}\right) / 2\right)$ and

$$
W_{s_{0}}(x)=\frac{1}{2 \pi i} \int_{(3)}(\pi x)^{-u} \frac{\Gamma\left(\frac{s_{0}+u+i T_{f}}{2}\right) \Gamma\left(\frac{s_{0}+u-i T_{f}}{2}\right)}{\Gamma\left(\frac{s_{0}+i T_{f}}{2}\right) \Gamma\left(\frac{s_{0}-i T_{f}}{2}\right)} e^{u^{2}} \frac{d u}{u} .
$$


The function $W_{s_{0}}(x)$ and its derivatives are bounded by

$$
\begin{aligned}
W_{s_{0}}(x) & \ll_{A} \min \left\{1,(x / \tau)^{-A}\right\}, \\
W_{s_{0}}^{(l)}(x) & \ll x^{-l} .
\end{aligned}
$$

The implied constants depend only on $\sigma_{0}, A$ and $l$.

\section{The proof of Theorem 1.1}

Hereafter $A$ and $B$ denote arbitrary large numbers, and $\epsilon$ represents an arbitrary small positive numbers. These values may be different at each occurrence (for example, we may sometimes replace $2 \epsilon$ with $\epsilon$ ). All the implied constants might be dependent on $A, B, \epsilon, A_{0}$ and Hecke-Maass form $f$. However, these implied constants are independent of the values of conductor $q$ and $\tau=\left|t_{0}\right|+3$. It follows from the approximate functional equations $(2.14),(2.18)$ that

$$
\begin{aligned}
& \sum_{\chi(\bmod q)}^{+} L\left(s_{0}, f \otimes \chi\right) \overline{L\left(s_{0}, \chi\right)} \\
= & \frac{1}{2} \sum_{\chi(\bmod q)}^{*}(\chi(-1)+1) \\
& \cdot\left(\sum_{n} \frac{\lambda_{f}(n) \chi(n)}{n^{s_{0}}} W_{s_{0}}\left(\frac{n}{q}\right)+\tau(\chi)^{2} q^{-2 s_{0}} \frac{\tilde{\gamma}\left(1-s_{0}\right)}{\tilde{\gamma}\left(s_{0}\right)} \sum_{n} \frac{\lambda_{f}(n) \bar{\chi}(n)}{n^{1-s_{0}}} W_{1-s_{0}}\left(\frac{n}{q}\right)\right) \\
& \cdot\left(\sum_{m} \frac{\bar{\chi}(m)}{m^{\overline{s_{0}}}} V_{\overline{s_{0}}}\left(\frac{m}{\sqrt{q}}\right)+\tau(\bar{\chi}) q^{-\overline{s_{0}}} \frac{\gamma\left(1-\overline{s_{0}}\right)}{\gamma\left(\overline{s_{0}}\right)} \sum_{m} \frac{\chi(m)}{m^{1-\overline{s_{0}}}} V_{1-\overline{s_{0}}}\left(\frac{m}{\sqrt{q}}\right)\right) \\
= & \frac{1}{2} \sum_{\chi(\bmod q)}^{*}(\chi(-1)+1)\left(S_{1}+S_{2}\right)\left(S_{3}+S_{4}\right),
\end{aligned}
$$

say. Hence it suffices to evaluate $\sum_{\chi(\bmod q)}^{*} \chi( \pm 1) S_{j} S_{k}$ for $j=1,2, k=3,4$.

3.1. The terms involving $\boldsymbol{S}_{\mathbf{1}} \boldsymbol{S}_{\mathbf{3}}$. We first evaluate the contribution of $S_{1} S_{3}$.

Lemma 3.1. We have

$$
\sum_{\chi(\bmod q)}^{*} \chi(-1) S_{1} S_{3} \ll(q \tau)^{\frac{3}{2}-\frac{3}{2} \sigma_{0}+\theta+\epsilon} .
$$

Proof. By definition and Lemma 2.1, we have

$$
\sum_{\chi(\bmod q)}^{*} \chi(-1) S_{1} S_{3}=(q-2) \Sigma_{1}-\Sigma_{2}
$$

where

$$
\begin{aligned}
\Sigma_{1}:= & \sum_{\substack{n \\
(n m, q)=1 \\
n+m \equiv 0(\bmod q)}} \frac{\lambda_{f}(n)}{n^{s_{0}}} W_{s_{0}}\left(\frac{n}{q}\right) \frac{1}{m^{\overline{s_{0}}}} V_{\overline{s_{0}}}\left(\frac{m}{\sqrt{q}}\right), \\
\Sigma_{2}:= & \sum_{\substack{n \\
(n m, q)=1 \\
n+m \neq 0(\bmod q)}} \frac{\lambda_{f}(n)}{n^{s_{0}}} W_{s_{0}}\left(\frac{n}{q}\right) \frac{1}{m^{\overline{s_{0}}}} V_{\overline{s_{0}}}\left(\frac{m}{\sqrt{q}}\right) .
\end{aligned}
$$


By Lemma 2.2 and the estimates (2.16), (2.20), the contribution of the terms with $n \geq(q \tau)^{1+\epsilon}$ to $\Sigma_{1}$ is at most

$$
\begin{aligned}
& \sum_{n \geq(q \tau)^{1+\epsilon}} \sum_{m} \frac{n^{\theta+\epsilon}}{(n m)^{\sigma_{0}}}\left(\frac{n}{q \tau}\right)^{-A}\left|V_{\overline{s_{0}}}\left(\frac{m}{\sqrt{q}}\right)\right| \\
& \ll(q \tau)^{A}\left((q \tau)^{1+\epsilon}\right)^{-A+\theta+\frac{1}{2}+\epsilon}\left(\sum_{m \leq(q \tau)^{\frac{1}{2}+\epsilon}} \frac{1}{m^{\sigma_{0}}}+\sum_{m \geq(q \tau)^{\frac{1}{2}+\epsilon}} \frac{1}{m^{\sigma_{0}}}\left(\frac{m}{\sqrt{q \tau}}\right)^{-A}\right) \\
& \ll(q \tau)^{-A} .
\end{aligned}
$$

Similarly, the contribution of the terms with $m \geq(q \tau)^{\frac{1}{2}+\epsilon}$ to $\Sigma_{1}$ is at most

$$
\begin{aligned}
& \sum_{n} \sum_{m \geq(q \tau)^{\frac{1}{2}+\epsilon}} \frac{n^{\theta+\epsilon}}{(n m)^{\sigma_{0}}}\left|W_{s_{0}}\left(\frac{n}{q}\right)\right|\left(\frac{m}{\sqrt{q \tau}}\right)^{-A} \\
& \ll\left(\sum_{n \leq(q \tau)^{1+\epsilon}} n^{\theta-\sigma_{0}+\epsilon}+\sum_{n \geq(q \tau)^{1+\epsilon}} n^{\theta-\sigma_{0}+\epsilon}\left(\frac{n}{q \tau}\right)^{-A}\right)(q \tau)^{\frac{A}{2}}\left((q \tau)^{\frac{1}{2}+\epsilon}\right)^{\frac{1}{2}-A} \\
& \ll(q \tau)^{-A} .
\end{aligned}
$$

Consequently it suffices to evaluate the contribution of the terms with $n \leq(q \tau)^{1+\epsilon}$, $m \leq(q \tau)^{\frac{1}{2}+\epsilon}$. By Lemma 2.2, the contribution of these terms to $(q-2) \Sigma_{1}$ is at most

$$
q(q \tau)^{\theta+\epsilon} \sum_{\substack{n \leq(q \tau)^{1+\epsilon} \\ n+m \equiv 0(\bmod q)}} \frac{1}{\substack{m \leq(q \tau)^{\frac{1}{2}+\epsilon} \\(n m)^{\sigma 0}}}
$$

Write $n=k q-m, \quad(m+1) / q \leq k \ll q^{\epsilon} \tau^{1+\epsilon}$. Then the double sum in (3.4) is

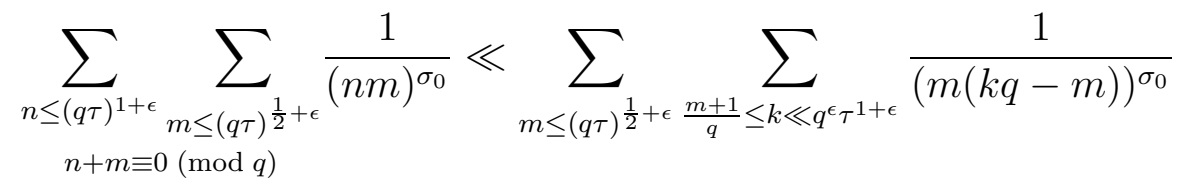

$$
\begin{aligned}
& \ll \sum_{m \leq(q \tau)^{\frac{1}{2}+\epsilon}} \sum_{1 \leq k \ll q^{\epsilon} \tau^{1+\epsilon}} \frac{1}{(k q m)^{\sigma_{0}}} \\
& \ll q^{\frac{1}{2}-\frac{3}{2} \sigma_{0}+\epsilon} \tau^{\frac{3}{2}-\frac{3}{2} \sigma_{0}+\epsilon} \text {. }
\end{aligned}
$$

Therefore, by (3.4) and (3.5), we have

$$
(q-2) \Sigma_{1} \ll(q \tau)^{\frac{3}{2}-\frac{3}{2} \sigma_{0}+\theta+\epsilon} .
$$

On the other hand, by Lemma 2.2, we have

$$
\Sigma_{2} \ll \sum_{n \leq(q \tau)^{1+\epsilon}} \frac{n^{\theta+\epsilon}}{n^{\sigma_{0}}} \sum_{m \leq(q \tau)^{\frac{1}{2}+\epsilon}} \frac{1}{m^{\sigma_{0}}} \ll(q \tau)^{\frac{3}{2}-\frac{3}{2} \sigma_{0}+\theta+\epsilon} .
$$

Substituting (3.6), (3.7) into (3.3), we obtain (3.2).

Next, we compute $\sum_{\chi(\bmod q)}^{*} S_{1} S_{3}$. This gives the main term in (1.2). 
Lemma 3.2. We have

$$
\sum_{\chi(\bmod q)}^{*} S_{1} S_{3}=L\left(2 \sigma_{0}, f\right) q+O\left(q^{1-2 \sigma_{0}+\epsilon} \tau^{\theta+\epsilon}+q^{\frac{1}{4}+\epsilon} \tau^{\frac{3}{2}-\frac{3}{2} \sigma_{0}}+(q \tau)^{1-\frac{\sigma_{0}}{2}+\theta+\epsilon}\right) .
$$

Proof. By Lemma 2.1, we have

$$
\sum_{\chi(\bmod q)}^{*} S_{1} S_{3}=(q-2) \Sigma_{3}-\Sigma_{4}
$$

where

$$
\begin{aligned}
\Sigma_{3} & :=\sum_{\substack{n \\
(n m, q)=1 \\
n \equiv m(\bmod q)}} \sum_{\substack{m \\
n \equiv}} \frac{\lambda_{f}(n)}{n^{s_{0}} m^{\overline{s_{0}}}} W_{s_{0}}\left(\frac{n}{q}\right) V_{\overline{s_{0}}}\left(\frac{m}{\sqrt{q}}\right), \\
\Sigma_{4}:= & \sum_{\substack{n \\
(n m, q)=1 \\
n \neq m(\bmod q)}} \sum_{m} \frac{\lambda_{f}(n)}{n^{s_{0}} m^{\bar{s} 0}} W_{s_{0}}\left(\frac{n}{q}\right) V_{\overline{s_{0}}}\left(\frac{m}{\sqrt{q}}\right) .
\end{aligned}
$$

In the same way as (3.7), we obtain

$$
\Sigma_{4} \ll(q \tau)^{\frac{3}{2}-\frac{3}{2} \sigma_{0}+\theta+\epsilon} .
$$

Next, we decompose $\Sigma_{3}$ by

$$
\Sigma_{3}=\Sigma_{3,1}+\Sigma_{3,2}
$$

where $\Sigma_{3,1}$ denotes the contribution of the terms with $n=m$, and $\Sigma_{3,2}$ denotes the contribution of remaining terms. We first compute $\Sigma_{3,1}$, which is given by

$$
\Sigma_{3,1}=\sum_{\substack{n=1 \\(n, q)=1}}^{\infty} \frac{\lambda_{f}(n)}{n^{2 \sigma_{0}}} W_{s_{0}}\left(\frac{n}{q}\right) V_{\overline{s_{0}}}\left(\frac{n}{\sqrt{q}}\right)
$$

We remove the condition $(n, q)=1$. Then the cost is at most

$$
\sum_{k=1}^{\infty} \frac{\lambda_{f}(q k)}{(q k)^{2 \sigma_{0}}} W_{s_{0}}(k) V_{\overline{s_{0}}}(\sqrt{q} k) \ll \sum_{k=1}^{\infty} \frac{(q k)^{\theta+\epsilon}}{(q k)^{2 \sigma_{0}}}\left(\frac{\sqrt{q} k}{\sqrt{\tau}}\right)^{-2 \theta-\epsilon} \ll q^{-2 \sigma_{0}+\epsilon} \tau^{\theta+\epsilon} .
$$

Here, we used the estimates $W_{s_{0}}(k) \ll 1, V_{\overline{s_{0}}}(\sqrt{q} k) \ll(\sqrt{q} k / \sqrt{\tau})^{-2 \theta-\epsilon}$. Thus we have

$$
\Sigma_{3,1}=\sum_{n=1}^{\infty} \frac{\lambda_{f}(n)}{n^{2 \sigma_{0}}} W_{s_{0}}\left(\frac{n}{q}\right) V_{\overline{s_{0}}}\left(\frac{n}{\sqrt{q}}\right)+O\left(q^{-2 \sigma_{0}+\epsilon} \tau^{\theta+\epsilon}\right)
$$


Substituting the definitions of the functions $W_{s_{0}}, V_{\overline{s_{0}}}$ and writing the $n$-sum as an $L$-function, we obtain

$$
\begin{aligned}
\sum_{n=1}^{\infty} \frac{\lambda_{f}(n)}{n^{2 \sigma_{0}}} W_{s_{0}}\left(\frac{n}{q}\right) V_{\overline{s_{0}}}\left(\frac{n}{\sqrt{q}}\right) \\
=\frac{1}{(2 \pi i)^{2}} \int_{(3)} \int_{(3)}\left(\frac{\pi}{q}\right)^{-z-\frac{1}{2} w} L\left(2 \sigma_{0}+z+w, f\right) \\
\quad \cdot \frac{\Gamma\left(\frac{s_{0}+z+i T_{f}}{2}\right) \Gamma\left(\frac{s_{0}+z-i T_{f}}{2}\right) \Gamma\left(\frac{\overline{s_{0}}+w}{2}\right)}{\Gamma\left(\frac{s_{0}+i T_{f}}{2}\right) \Gamma\left(\frac{s_{0}-i T_{f}}{2}\right) \Gamma\left(\frac{\overline{s_{0}}}{2}\right)} e^{z^{2}+w^{2}} \frac{d z}{z} \frac{d w}{w} .
\end{aligned}
$$

We move the lines of integrals to $\Re(z)=\Re(w)=-1 / 2+\epsilon$. Then we cross a pole at $z=w=0$ and its residue is $L\left(2 \sigma_{0}, f\right)$. To evaluate the new integral, we use the upper bound

$$
\frac{\Gamma(s+z)}{\Gamma(s)} \ll_{\sigma, \beta} \frac{|s+z|^{\sigma+\beta-\frac{1}{2}}}{|s|^{\sigma-\frac{1}{2}}} \exp \left(\frac{\pi}{2}(|s|-|s+z|)\right) \quad(\Re(s)=\sigma>0, \Re(z)=\beta>-\sigma)
$$

(see [9], p. 100). Hence it follows that for $\Re(z)=\Re(w)=-1 / 2+\epsilon$, we have

$$
\begin{aligned}
& \frac{\Gamma\left(\frac{s_{0}+z \pm i T_{f}}{2}\right)}{\Gamma\left(\frac{s_{0} \pm i T_{f}}{2}\right)} \ll f \tau^{\frac{1}{2}-\frac{\sigma_{0}}{2}}\left|s_{0}+z\right|^{\frac{\sigma_{0}}{2}-\frac{3}{4}+\epsilon} \exp \left(\frac{\pi}{4}\left(\left|s_{0}\right|-\left|s_{0}+z\right|\right)\right) \\
& \leq \tau^{\frac{1}{2}-\frac{\sigma_{0}}{2}}\left|s_{0}+z\right|^{\frac{\sigma_{0}}{2}-\frac{3}{4}+\epsilon} e^{\frac{\pi|z|}{4}}, \\
& \frac{\Gamma\left(\frac{\overline{s_{0}}+w}{2}\right)}{\Gamma\left(\frac{\overline{s_{0}}}{2}\right)} \ll \tau^{\frac{1}{2}-\frac{\sigma_{0}}{2}}\left|\overline{s_{0}}+w\right|^{\frac{\sigma_{0}}{2}-\frac{3}{4}+\epsilon} \exp \left(\frac{\pi}{4}\left(\left|\overline{s_{0}}\right|-\left|\overline{s_{0}}+w\right|\right)\right) \\
& \leq \tau^{\frac{1}{2}-\frac{\sigma_{0}}{2}}\left|\overline{s_{0}}+w\right|^{\frac{\sigma_{0}}{2}-\frac{3}{4}+\epsilon} e^{\frac{\pi|w|}{4}} .
\end{aligned}
$$

On the other hand, since $\left(1-\Re\left(2 \sigma_{0}+z+w\right)\right) / 2=1-\sigma_{0}-\epsilon \leq 1 / 2-\epsilon$, by the convexity bound for $L$-function we obtain

$$
L\left(2 \sigma_{0}+z+w, f\right) \ll_{f}\left(\left|2 \sigma_{0}+z+w\right|^{2}+3\right)^{\frac{1}{2}-\epsilon}
$$

(see [9], p. 100, (5.20)). Combining these estimates we find that

$$
\begin{aligned}
& \frac{1}{(2 \pi i)^{2}} \int_{\left(-\frac{1}{2}+\epsilon\right)} \int_{\left(-\frac{1}{2}+\epsilon\right)}\left(\frac{\pi}{q}\right)^{-z-\frac{1}{2} w} L\left(2 \sigma_{0}+z+w, f\right) \\
& \quad \frac{\Gamma\left(\frac{s_{0}+z+i T_{f}}{2}\right) \Gamma\left(\frac{s_{0}+z-i T_{f}}{2}\right) \Gamma\left(\frac{\overline{s_{0}}+w}{2}\right)}{\Gamma\left(\frac{s_{0}+i T_{f}}{2}\right) \Gamma\left(\frac{s_{0}-i T_{f}}{2}\right) \Gamma\left(\frac{\bar{s}_{0}}{2}\right)} e^{z^{2}+w^{2}} \frac{d z}{z} \frac{d w}{w} \\
& \ll q^{-\frac{3}{4}+\epsilon} \tau^{\frac{3}{2}-\frac{3}{2} \sigma_{0}} .
\end{aligned}
$$

By (3.12) and (3.13), we have

$$
\Sigma_{3,1}=L\left(2 \sigma_{0}, f\right)+O\left(q^{-2 \sigma_{0}+\epsilon} \tau^{\theta+\epsilon}+q^{-\frac{3}{4}+\epsilon} \tau^{\frac{3}{2}-\frac{3}{2} \sigma_{0}}\right) .
$$


Next we evaluate the off-diagonal term

$$
\Sigma_{3,2}:=\sum_{\substack{n \\ n \equiv m(\bmod q) \\ n \neq m,(n m, q)=1}} \frac{\lambda_{f}(n)}{n^{s_{0}} m^{\overline{s_{0}}}} W_{s_{0}}\left(\frac{n}{q}\right) V_{\overline{s_{0}}}\left(\frac{m}{\sqrt{q}}\right) .
$$

By the same argument as we discussed in the proof of Lemma 3.1, we find that the contribution of the terms with $n>(q \tau)^{1+\epsilon}$ or $m>(q \tau)^{\frac{1}{2}+\epsilon}$ to $\Sigma_{3,2}$ is $O\left((q \tau)^{-A}\right)$. Hence by Lemma 2.2, we have

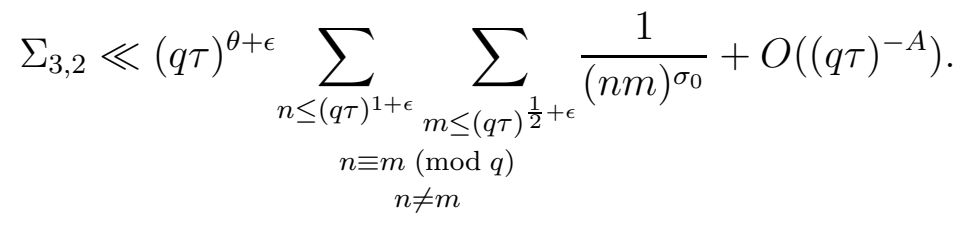

Let us evaluate the sum of the right hand side of (3.15). We first evaluate the contribution of the terms with $n<m$. Write $n=m-q k, 1 \leq k<m / q$. Then,

$$
\begin{aligned}
\sum_{\substack{n \leq(q \tau)^{1+\epsilon} \\
n \equiv m(\bmod q) \\
n<m}} \sum_{\substack{m \leq(q))^{\frac{1}{2}+\epsilon} \\
n<m}} \frac{1}{(n m)^{\sigma_{0}}} & =\sum_{\substack{q<m \leq(q \tau)^{\frac{1}{2}+\epsilon} \\
1 \leq k<\frac{m}{q}}} \frac{1}{(m(m-q k))^{\sigma_{0}}} \\
& \ll \sum_{m \leq(q \tau)^{\frac{1}{2}+\epsilon}} \sum_{1 \leq k<\frac{m}{q}} \frac{1}{m^{\sigma_{0}}} \\
& \ll q^{-1}(q \tau)^{1-\frac{\sigma_{0}}{2}+\epsilon} .
\end{aligned}
$$

On the other hand, if $n>m$, we have the expression $n=m+q k, 1 \leq k \ll q^{\epsilon} \tau^{1+\epsilon}$. Hence

$$
\begin{aligned}
\sum_{\substack{n \leq(q \tau)^{1+\epsilon} \\
n \equiv m(\bmod q) \\
n>m}} \sum_{\substack{m \leq(q \tau) \\
\frac{1}{2}+\epsilon}} \frac{1}{(n m)^{\sigma_{0}}} & \ll \sum_{m \leq(q \tau)^{\frac{1}{2}+\epsilon}} \sum_{1 \leq k \ll q^{\epsilon} \tau^{1+\epsilon}} \frac{1}{(m(m+q k))^{\sigma_{0}}} \\
& \ll q^{-\sigma_{0}} \sum_{m \leq(q \tau)^{\frac{1}{2}+\epsilon}} m^{-\sigma_{0}} \sum_{1 \leq k \ll q^{\epsilon} \tau^{1+\epsilon}} k^{-\sigma_{0}} \\
& \ll q^{-1}(q \tau)^{\frac{3}{2}-\frac{3}{2} \sigma_{0}+\epsilon} .
\end{aligned}
$$

Note that since $\sigma_{0} \geq 1 / 2$, the right hand side of (3.17) is dominated by the right hand side of (3.16). Hence we have

$$
\sum_{\substack{n \leq(q \tau)^{1+\epsilon} \\ n \equiv m(\bmod q) \\ n \neq m}} \sum_{\substack{m \leq(q \tau)^{\frac{1}{2}+\epsilon} \\ n \neq m}} \frac{1}{(n m)^{\sigma_{0}}} \ll q^{-1}(q \tau)^{1-\frac{\sigma_{0}}{2}+\epsilon} .
$$

By (3.15) and (3.18), we have

$$
\Sigma_{3,2} \ll q^{-1}(q \tau)^{1-\frac{\sigma_{0}}{2}+\theta+\epsilon} .
$$

Combining (3.14) and (3.19), we have

$$
\Sigma_{3}=L\left(2 \sigma_{0}, f\right)+O\left(q^{-2 \sigma_{0}+\epsilon} \tau^{\theta+\epsilon}+q^{-\frac{3}{4}+\epsilon} \tau^{\frac{3}{2}-\frac{3}{2} \sigma_{0}}+q^{-1}(q \tau)^{1-\frac{\sigma_{0}}{2}+\theta+\epsilon}\right) .
$$

Finally, by substituting (3.10) and (3.20) into (3.9), we obtain (3.8). 
By (3.2) and (3.8), we obtain

$$
\begin{aligned}
& \sum_{\chi(\bmod q)}^{*}(\chi(-1)+1) S_{1} S_{3} \\
& \quad=L\left(2 \sigma_{0}, f\right) q+O\left(q^{1-\theta-2 \sigma_{0}+\epsilon}(q \tau)^{\theta+\epsilon}+q^{-\frac{5}{4}+\frac{3}{2} \sigma_{0}+\epsilon}(q \tau)^{\frac{3}{2}-\frac{3}{2} \sigma_{0}}+(q \tau)^{1-\frac{\sigma_{0}}{2}+\theta+\epsilon}\right) .
\end{aligned}
$$

\subsection{The terms involving $S_{2} S_{4}$.}

Lemma 3.3. We have

$$
\sum_{\chi(\bmod q)}^{*}(\chi(-1)+1) S_{2} S_{4} \ll q^{-\frac{1}{2}}(q \tau)^{-\frac{3}{2} \sigma_{0}+2+\epsilon} .
$$

Proof. By definition we have

$$
\begin{aligned}
& \sum_{\chi(\bmod q)}^{*}(\chi(-1)+1) S_{2} S_{4} \\
= & q^{-2 s_{0}-\overline{s_{0}}} \frac{\tilde{\gamma}\left(1-s_{0}\right) \gamma\left(1-\overline{s_{0}}\right)}{\tilde{\gamma}\left(s_{0}\right) \gamma\left(\overline{s_{0}}\right)} \sum_{\chi(\bmod q)}^{*}(\chi(-1)+1) \tau(\chi)^{2} \tau(\bar{\chi}) \\
& \cdot \sum_{n} \frac{\lambda_{f}(n) \bar{\chi}(n)}{n^{1-s_{0}}} W_{1-s_{0}}\left(\frac{n}{q}\right) \sum_{m} \frac{\chi(m)}{m^{1-\overline{s_{0}}}} V_{1-\overline{s_{0}}}\left(\frac{m}{\sqrt{q}}\right) .
\end{aligned}
$$

Since

$$
\tau(\chi) \tau(\bar{\chi})=\tau(\chi) \overline{\tau(\chi)}=|\tau(\chi)|^{2}=q
$$

holds for even characters $\chi$, it follows that

$$
\begin{aligned}
& \sum_{\chi(\bmod q)}^{*}(\chi(-1)+1) S_{2} S_{4} \\
= & q^{1-2 s_{0}-\overline{s_{0}}} \frac{\tilde{\gamma}\left(1-s_{0}\right) \gamma\left(1-\overline{s_{0}}\right)}{\tilde{\gamma}\left(s_{0}\right) \gamma\left(\overline{s_{0}}\right)} \sum_{\chi(\bmod q)}^{*}(\chi(-1)+1) \tau(\chi) \\
& \cdot \sum_{n} \frac{\lambda_{f}(n) \bar{\chi}(n)}{n^{1-s_{0}}} W_{1-s_{0}}\left(\frac{n}{q}\right) \sum_{m} \frac{\chi(m)}{m^{1-s_{0}}} V_{1-\overline{s_{0}}}\left(\frac{m}{\sqrt{q}}\right) .
\end{aligned}
$$

First, by Stirling's approximation the quotient of gamma factors is evaluated by $O\left(\tau^{\frac{3}{2}-3 \sigma_{0}}\right)$ (see [9], (5.115)). Hence it suffices to evaluate

$$
\sum_{n} \frac{\lambda_{f}(n)}{n^{1-s_{0}}} W_{1-s_{0}}\left(\frac{n}{q}\right) \sum_{m} \frac{1}{m^{1-\overline{s_{0}}}} V_{1-\overline{s_{0}}}\left(\frac{m}{\sqrt{q}}\right) \sum_{\chi(\bmod q)}^{*} \tau(\chi) \bar{\chi}( \pm n) \chi(m) .
$$

Since the Gauss sum $\tau(\chi)$ is defined by

$$
\tau(\chi)=\sum_{a(\bmod q)}^{*} \chi(a) e\left(\frac{a}{q}\right)
$$

the innermost sum in (3.25) becomes

$$
\sum_{\chi(\bmod q)}^{*}\left(\sum_{a(\bmod q)}^{*} \chi(a) e\left(\frac{a}{q}\right)\right) \bar{\chi}( \pm n) \chi(m)=\sum_{a(\bmod q)}^{*} e\left(\frac{a}{q}\right) \sum_{\chi(\bmod q)}^{*} \bar{\chi}( \pm n) \chi(a m) .
$$


Using this and Lemma 2.1, it follows that (3.25) is equal to

$$
\begin{aligned}
& (q-2) \sum_{a(\bmod q)}^{*} e\left(\frac{a}{q}\right) \sum_{\substack{n \\
\pm n \equiv a m(\bmod q) \\
(n m, q)=1}} \frac{\lambda_{f}(n)}{n^{1-s_{0}}} W_{1-s_{0}}\left(\frac{n}{q}\right) \frac{1}{m^{1-\overline{s_{0}}}} V_{1-\overline{s_{0}}}\left(\frac{m}{\sqrt{q}}\right) \\
& -\sum_{a(\bmod q)}^{*} e\left(\frac{a}{q}\right) \sum_{\substack{n \\
\pm n \neq a m(\bmod q) \\
(n m, q)=1}} \frac{\lambda_{f}(n)}{n^{1-s_{0}}} W_{1-s_{0}}\left(\frac{n}{q}\right) \frac{1}{m^{1-\overline{s_{0}}}} V_{1-\overline{s_{0}}}\left(\frac{m}{\sqrt{q}}\right) \\
& =(q-1) \sum_{a(\bmod q)}^{*} e\left(\frac{a}{q}\right) \sum_{\substack{n \\
\pm n \equiv a m(\bmod q) \\
(n m, q)=1}} \sum_{\substack{m \\
n^{1-s_{0}}}} W_{1-s_{0}}\left(\frac{n}{q}\right) \frac{1}{m^{1-\overline{s_{0}}}} V_{1-\overline{s_{0}}}\left(\frac{m}{\sqrt{q}}\right) \\
& -\sum_{\substack{n \\
(n m, q)=1}} \sum_{m} \frac{\lambda_{f}(n)}{n^{1-s_{0}}} W_{1-s_{0}}\left(\frac{n}{q}\right) \frac{1}{m^{1-\overline{s_{0}}}} V_{1-\overline{s_{0}}}\left(\frac{m}{\sqrt{q}}\right) \sum_{a(\bmod q)}^{*} e\left(\frac{a}{q}\right) \\
& =(q-1) \sum_{\substack{n \\
(n m, q)=1}} \sum_{m} \frac{\lambda_{f}(n)}{n^{1-s_{0}}} W_{1-s_{0}}\left(\frac{n}{q}\right) \frac{1}{m^{1-\overline{s_{0}}}} V_{1-\overline{s_{0}}}\left(\frac{m}{\sqrt{q}}\right) e\left(\frac{ \pm n \bar{m}}{q}\right) \\
& -\sum_{\substack{n \\
(n m, q)=1}} \sum_{m} \frac{\lambda_{f}(n)}{n^{1-s_{0}}} W_{1-s_{0}}\left(\frac{n}{q}\right) \frac{1}{m^{1-\overline{s 0}}} V_{1-\overline{s_{0}}}\left(\frac{m}{\sqrt{q}}\right) \sum_{a(\bmod q)}^{*} e\left(\frac{a}{q}\right) .
\end{aligned}
$$

We denote the last two lines by

$$
(q-1) \Sigma_{5}-\Sigma_{6}
$$

We first evaluate $\Sigma_{6}$. Since

$$
\sum_{a(\bmod q)}^{*} e\left(\frac{a}{q}\right)=-1
$$

we have

$$
\Sigma_{6} \ll(q \tau)^{\theta+\epsilon} \sum_{n \leq(q \tau)^{1+\epsilon}} \frac{1}{n^{1-\sigma_{0}}} \sum_{m \leq(q \tau)^{\frac{1}{2}+\epsilon}} \frac{1}{m^{1-\sigma_{0}}} \ll(q \tau)^{\frac{3}{2} \sigma_{0}+\theta+\epsilon} .
$$

Next, we evaluate $\Sigma_{5}$. We first remove the condition $(n, q)=1$. Then the cost is at most

$$
\begin{aligned}
& \sum_{n^{\prime}=1}^{\infty} \frac{\left(q n^{\prime}\right)^{\theta+\epsilon}}{\left(q n^{\prime}\right)^{1-\sigma_{0}}}\left|W_{1-s_{0}}\left(n^{\prime}\right)\right| \sum_{m} \frac{1}{m^{1-\sigma_{0}}}\left|V_{1-\overline{s_{0}}}\left(\frac{m}{\sqrt{q}}\right)\right| \\
& \ll q^{-1+\sigma_{0}+\theta+\epsilon}\left(\sum_{n^{\prime} \leq \tau^{1+\epsilon}} n^{\prime-1+\theta+\sigma_{0}}\right)\left(\sum_{m \leq(q \tau)^{\frac{1}{2}+\epsilon}} m^{-1+\sigma_{0}}\right) \\
& \ll q^{-1}(q \tau)^{\frac{3}{2} \sigma_{0}+\theta+\epsilon} .
\end{aligned}
$$


Hence we get

$$
\begin{aligned}
\Sigma_{5}= & \sum_{\substack{m \\
(m, q)=1}} \frac{1}{m^{1-\overline{s_{0}}}} V_{1-\overline{s_{0}}}\left(\frac{m}{\sqrt{q}}\right) \sum_{n} \frac{\lambda_{f}(n)}{n^{1-s_{0}}} W_{1-s_{0}}\left(\frac{n}{q}\right) e\left(\frac{ \pm n \bar{m}}{q}\right) \\
& +O\left(q^{-1}(q \tau)^{\frac{3}{2} \sigma_{0}+\theta+\epsilon}\right) .
\end{aligned}
$$

By partial summation, the $n$-sum in (3.28) is

$$
\begin{aligned}
& \sum_{n} \frac{\lambda_{f}(n)}{n^{1-s_{0}}} W_{1-s_{0}}\left(\frac{n}{q}\right) e\left(\frac{ \pm n \bar{m}}{q}\right) \\
& \sim \sum_{n \leq(q \tau)^{1+\epsilon}} \frac{\lambda_{f}(n)}{n^{1-s_{0}}} W_{1-s_{0}}\left(\frac{n}{q}\right) e\left(\frac{ \pm n \bar{m}}{q}\right) \\
& =\int_{1}^{(q \tau)^{1+\epsilon}} u^{-1+s_{0}} W_{1-s_{0}}\left(\frac{u}{q}\right) d\left(\sum_{n \leq u} \lambda_{f}(n) e\left(\frac{ \pm \bar{m} n}{q}\right)\right) \\
& =\left[u^{-1+s_{0}} W_{1-s_{0}}\left(\frac{u}{q}\right) \sum_{n \leq u} \lambda_{f}(n) e\left(\frac{ \pm \bar{m} n}{q}\right)\right]_{u=1}^{(q \tau)^{1+\epsilon}} \\
& \quad-\int_{1}^{(q \tau)^{1+\epsilon}}\left(\sum_{n \leq u} \lambda_{f}(n) e\left(\frac{ \pm \bar{m} n}{q}\right)\right) \frac{d}{d u}\left(u^{-1+s_{0}} W_{1-s_{0}}\left(\frac{u}{q}\right)\right) d u .
\end{aligned}
$$

Using the bounds $W_{1-s_{0}}(x) \ll 1, W_{1-s_{0}}^{\prime}(x) \ll x^{-1}$ and Lemma 2.5, it follow that the above is $O\left(q^{-1}(q \tau)^{\sigma_{0}+\frac{1}{2}+\epsilon}\right)$. On the other hand, the $m$-sum in (3.28) is at most

$$
\sum_{m \leq(q \tau)^{\frac{1}{2}+\epsilon}} \frac{1}{m^{1-\sigma_{0}}} \ll(q \tau)^{\frac{\sigma_{0}}{2}+\epsilon}
$$

Consequently the first term of the right hand side of $(3.28)$ is $O\left(q^{-1}(q \tau)^{\frac{3}{2} \sigma_{0}+\frac{1}{2}+\epsilon}\right)$. Note that the $O$ - term in (3.28) is dominated by this, because $\theta<1 / 2$. Therefore we have

$$
\Sigma_{5} \ll q^{-1}(q \tau)^{\frac{3}{2} \sigma_{0}+\frac{1}{2}+\epsilon}
$$

Substituting (3.27), (3.29) into (3.26), we find that (3.25) is $O\left((q \tau)^{\frac{3}{2} \sigma_{0}+\frac{1}{2}+\epsilon}\right)$. Combining this with the estimate of the quotient of gamma factors and the trivial estimate $q^{1-2 s_{0}-\overline{s_{0}}} \ll q^{1-3 \sigma_{0}}$, we obtain (3.22).

\subsection{The terms involving $S_{1} S_{4}$.}

Lemma 3.4. We have

$$
\sum_{\chi(\bmod q)}^{*}(\chi(-1)+1) S_{1} S_{4} \ll q^{-\frac{1}{2}}(q \tau)^{\frac{3}{2}-\frac{\sigma_{0}}{2}+\epsilon}
$$


Proof. By definition we have

$$
\begin{aligned}
& \sum_{\chi(\bmod q)}^{*}(\chi(-1)+1) S_{1} S_{4} \\
= & q^{-\overline{s_{0}}} \frac{\gamma\left(1-\overline{s_{0}}\right)}{\gamma\left(\overline{s_{0}}\right)} \sum_{\chi(\bmod q)}^{*}(\chi(-1)+1) \\
& \cdot \sum_{n} \frac{\lambda_{f}(n) \chi(n)}{n^{s_{0}}} W_{s_{0}}\left(\frac{n}{q}\right) \tau(\bar{\chi}) \sum_{m} \frac{\chi(m)}{m^{1-\overline{s_{0}}}} V_{1-\overline{s_{0}}}\left(\frac{m}{\sqrt{q}}\right) .
\end{aligned}
$$

The quotient of gamma factors is $O\left(\tau^{\frac{1}{2}-\sigma_{0}}\right)$. Hence it suffices to evaluate

$$
\sum_{n} \frac{\lambda_{f}(n)}{n^{s_{0}}} W_{s_{0}}\left(\frac{n}{q}\right) \sum_{m} \frac{1}{m^{1-\overline{s_{0}}}} V_{1-\overline{s_{0}}}\left(\frac{m}{\sqrt{q}}\right) \sum_{\chi(\bmod q)}^{*} \tau(\bar{\chi}) \chi( \pm n) \chi(m) .
$$

Since the Gauss sum is given by

$$
\tau(\bar{\chi})=\sum_{a(\bmod q)}^{*} \bar{\chi}(a) e\left(\frac{a}{q}\right)
$$

we get

$$
\sum_{\chi(\bmod q)}^{*} \tau(\bar{\chi}) \chi( \pm n) \chi(m)=\sum_{a(\bmod q)}^{*} e\left(\frac{a}{q}\right) \sum_{\chi(\bmod q)}^{*} \chi( \pm n m) \bar{\chi}(a) .
$$

Hence by Lemma 2.1, we find that (3.32) becomes

$$
(q-1) \Sigma_{7}-\Sigma_{8}
$$

where

$$
\begin{aligned}
& \Sigma_{7}=\sum_{\substack{n \\
(n m, q)=1}} \sum_{m} e\left(\frac{ \pm n m}{q}\right) \frac{\lambda_{f}(n)}{n^{s_{0}}} W_{s_{0}}\left(\frac{n}{q}\right) \frac{1}{m^{1-\overline{s_{0}}}} V_{1-\overline{s_{0}}}\left(\frac{m}{\sqrt{q}}\right), \\
& \Sigma_{8}=\sum_{a(\bmod q)}^{*} e\left(\frac{a}{q}\right) \sum_{\substack{n \\
(n m, q)=1}} \sum_{m} \frac{\lambda_{f}(n)}{n^{s_{0}}} W_{s_{0}}\left(\frac{n}{q}\right) \frac{1}{m^{1-\overline{s_{0}}}} V_{1-\overline{s_{0}}}\left(\frac{m}{\sqrt{q}}\right) .
\end{aligned}
$$

We first evaluate $\Sigma_{8}$. Since the $a$-sum is equal to -1 , by Lemma 2.2 , we have

$$
\begin{aligned}
\Sigma_{8} & \ll \sum_{n} \frac{\left|\lambda_{f}(n)\right|}{n^{\sigma_{0}}}\left|W_{s_{0}}\left(\frac{n}{q}\right)\right| \sum_{m} \frac{1}{m^{1-\sigma_{0}}}\left|V_{1-\overline{s_{0}}}\left(\frac{m}{\sqrt{q}}\right)\right| \\
& \ll(q \tau)^{\theta+\epsilon} \sum_{n \leq(q \tau)^{1+\epsilon}} \frac{1}{n^{\sigma_{0}}} \sum_{m \leq(q \tau)^{\frac{1}{2}+\epsilon}} \frac{1}{m^{1-\sigma_{0}}} \\
& \ll(q \tau)^{1-\frac{\sigma_{0}}{2}+\theta+\epsilon} .
\end{aligned}
$$

Next, we evaluate $\Sigma_{7}$. We remove the condition $(n, q)=1$. Then the cost is at most

$$
\begin{aligned}
\sum_{n^{\prime}} \frac{\left(q n^{\prime}\right)^{\theta+\epsilon}}{\left(q n^{\prime}\right)^{\sigma_{0}}}\left|W_{s_{0}}\left(n^{\prime}\right)\right| \sum_{m \leq(q \tau)^{\frac{1}{2}+\epsilon}} \frac{1}{m^{1-\sigma_{0}}} & \ll q^{\theta-\sigma_{0}+\epsilon}\left(\sum_{n^{\prime} \leq \tau^{1+\epsilon}} n^{\prime \theta-\sigma_{0}+\epsilon}\right)(q \tau)^{\frac{\sigma_{0}}{2}+\epsilon} \\
& \ll \tau^{1+\epsilon}(q \tau)^{\theta-\frac{\sigma_{0}}{2}+\epsilon} .
\end{aligned}
$$


Hence

$$
\begin{aligned}
\Sigma_{7}= & \sum_{\substack{m \\
(m, q)=1}} \frac{1}{m^{1-\overline{s_{0}}}} V_{1-\overline{s_{0}}}\left(\frac{m}{\sqrt{q}}\right) \sum_{n} e\left(\frac{ \pm n m}{q}\right) \frac{\lambda_{f}(n)}{n^{s_{0}}} W_{s_{0}}\left(\frac{n}{q}\right) \\
& +O\left(\tau^{1+\epsilon}(q \tau)^{\theta-\frac{\sigma_{0}}{2}+\epsilon}\right) .
\end{aligned}
$$

By partial summation, the $n$-sum in (3.35) is

$$
\begin{aligned}
\sum_{n} e\left(\frac{ \pm n m}{q}\right) \frac{\lambda_{f}(n)}{n^{s_{0}}} W_{s_{0}}\left(\frac{n}{q}\right) & \sim \sum_{n \leq(q \tau)^{1+\epsilon}} e\left(\frac{ \pm n m}{q}\right) \frac{\lambda_{f}(n)}{n^{s_{0}}} W_{s_{0}}\left(\frac{n}{q}\right) \\
= & \int_{1}^{(q \tau)^{1+\epsilon}} u^{-s_{0}} W_{s_{0}}\left(\frac{u}{q}\right) d\left(\sum_{n \leq u} \lambda_{f}(n) e\left( \pm \frac{n m}{q}\right)\right) \\
= & {\left[u^{-s_{0}} W_{s_{0}}\left(\frac{u}{q}\right) \sum_{n \leq u} \lambda_{f}(n) e\left( \pm \frac{n m}{q}\right)\right]_{u=1}^{(q \tau)^{1+\epsilon}} } \\
& -\int_{1}^{(q \tau)^{1+\epsilon}}\left(\sum_{n \leq u} \lambda_{f}(n) e\left( \pm \frac{n m}{q}\right)\right) \frac{d}{d u}\left(u^{-s_{0}} W_{s_{0}}\left(\frac{u}{q}\right)\right) d u .
\end{aligned}
$$

Using the bounds $W_{s_{0}}(x) \ll 1, W_{s_{0}}^{\prime}(x) \ll x^{-1}$ and Lemma 2.5, it follows that the above is $O\left(\tau(q \tau)^{\epsilon}\right)$. Consequently we have

$$
\Sigma_{7} \ll \tau(q \tau)^{\epsilon} \sum_{m \leq(q \tau)^{\frac{1}{2}+\epsilon}} \frac{1}{m^{1-\sigma_{0}}}+O\left(\tau^{1+\epsilon}(q \tau)^{\theta-\frac{\sigma_{0}}{2}+\epsilon}\right) \ll \tau(q \tau)^{\frac{\sigma_{0}}{2}+\epsilon} .
$$

Here, we used $\theta<1 / 2$. Substituting (3.34), (3.36) into (3.33), we find that (3.32) becomes $O\left((q \tau)^{1+\frac{\sigma_{0}}{2}+\epsilon}\right)$. Combining this with the estimate of the quotient of gamma factors and the trivial estimate $q^{-\overline{s_{0}}} \ll q^{-\sigma_{0}}$, we obtain (3.30).

3.4. The terms involving $\boldsymbol{S}_{\mathbf{2}} \boldsymbol{S}_{\mathbf{3}}$. Finally we evaluate the terms involving $S_{2} S_{3}$.

Lemma 3.5. We have

$$
\begin{gathered}
\sum_{\chi(\bmod q)}^{*}(\chi(-1)+1) S_{2} S_{3} \ll q^{\frac{1}{2}}(q \tau)^{\frac{5}{4}+\frac{15}{26} \theta-\frac{87}{52} \sigma_{0}+\epsilon}+q^{-1}(q \tau)^{\frac{5}{2}-\frac{5}{4} \sigma_{0}+\epsilon} \\
+q^{-1}(q \tau)^{-\frac{87}{52} \sigma_{0}+\frac{37}{26} \theta+\frac{139}{52}+\epsilon} .
\end{gathered}
$$

Proof. By definition, we have

$$
\begin{aligned}
& \sum_{\chi(\bmod q)}^{*}(\chi(-1)+1) S_{2} S_{3} \\
= & q^{-2 s_{0}} \frac{\tilde{\gamma}\left(1-s_{0}\right)}{\tilde{\gamma}\left(s_{0}\right)} \sum_{\chi(\bmod q)}^{*}(\chi(-1)+1) \tau(\chi)^{2} \sum_{n} \frac{\lambda_{f}(n) \bar{\chi}(n)}{n^{1-s_{0}}} W_{1-s_{0}}\left(\frac{n}{q}\right) \\
& \cdot \sum_{m} \frac{\bar{\chi}(m)}{m^{\bar{s}}} V_{\overline{s_{0}}}\left(\frac{m}{\sqrt{q}}\right) .
\end{aligned}
$$


The quotient of gamma factors is $O\left(\tau^{1-2 \sigma_{0}}\right)$. Hence it suffices to evaluate

$$
\begin{aligned}
& q^{-2 s_{0}} \sum_{\chi(\bmod q)}^{*} \tau(\chi)^{2} \sum_{n} \frac{\lambda_{f}(n) \bar{\chi}( \pm n)}{n^{1-s_{0}}} W_{1-s_{0}}\left(\frac{n}{q}\right) \mathcal{W}_{1}\left(\frac{n}{N}\right) \\
& \cdot \sum_{m} \frac{\bar{\chi}(m)}{m^{\bar{s}}} V_{\overline{s_{0}}}\left(\frac{m}{\sqrt{q}}\right) \mathcal{W}_{2}\left(\frac{m}{M}\right)
\end{aligned}
$$

for $N<(q \tau)^{1+\epsilon}, M<(q \tau)^{\frac{1}{2}+\epsilon}$, where $\mathcal{W}_{1}$ and $\mathcal{W}_{2}$ are arbitrary smooth functions compactly supported in the interval $[1,2]$. Since

$$
\tau(\chi)=\sum_{a(\bmod q)}^{*} \chi(a) e\left(\frac{a}{q}\right)
$$

(3.39) is equal to

$$
\begin{aligned}
& q^{-2 s_{0}} \sum_{n} \frac{\lambda_{f}(n)}{n^{1-s_{0}}} W_{1-s_{0}}\left(\frac{n}{q}\right) \mathcal{W}_{1}\left(\frac{n}{N}\right) \sum_{m} \frac{1}{m^{\bar{s}}} V_{\overline{s_{0}}}\left(\frac{m}{\sqrt{q}}\right) \mathcal{W}_{2}\left(\frac{m}{M}\right) \\
& \cdot \sum_{\chi(\bmod q)}^{*}\left(\sum_{a, b(\bmod q)}^{*} \chi(a b) e\left(\frac{a+b}{q}\right)\right) \bar{\chi}( \pm n m) .
\end{aligned}
$$

By Lemma 2.1, this equals

$$
q^{-2 s_{0}}(q-1) \Sigma_{9}-q^{-2 s_{0}} \Sigma_{10}
$$

where

$$
\begin{aligned}
& \Sigma_{9}=\sum_{a, b(\bmod q)}^{*} e\left(\frac{a+b}{q}\right) \\
& \sum_{\substack{n \\
\pm n m \equiv a b(\bmod q) \\
(n m, q)=1}} \frac{\lambda_{f}(n)}{n^{1-s_{0}}} W_{1-s_{0}}\left(\frac{n}{q}\right) \mathcal{W}_{1}\left(\frac{n}{N}\right) \frac{1}{m^{\bar{s}}} V_{\overline{s_{0}}}\left(\frac{m}{\sqrt{q}}\right) \mathcal{W}_{2}\left(\frac{m}{M}\right) \\
& =\sum_{\substack{n \\
(n m, q)=1}} \sum_{m} \frac{\lambda_{f}(n)}{n^{1-s_{0}}} W_{1-s_{0}}\left(\frac{n}{q}\right) \mathcal{W}_{1}\left(\frac{n}{N}\right) \frac{1}{m^{\overline{s_{0}}}} V_{\overline{s_{0}}}\left(\frac{m}{\sqrt{q}}\right) \mathcal{W}_{2}\left(\frac{m}{M}\right) \\
& \sum_{a(\bmod q)}^{*} e\left(\frac{a \pm \bar{a} n m}{q}\right) \\
& \Sigma_{10}=\sum_{\substack{n \\
(n m, q)=1}} \sum_{m} \frac{\lambda_{f}(n)}{n^{1-s_{0}}} W_{1-s_{0}}\left(\frac{n}{q}\right) \mathcal{W}_{1}\left(\frac{n}{N}\right) \frac{1}{m^{\overline{s_{0}}}} V_{\overline{s_{0}}}\left(\frac{m}{\sqrt{q}}\right) \mathcal{W}_{2}\left(\frac{m}{M}\right) \sum_{a, b(\bmod q)}^{*} e\left(\frac{a+b}{q}\right) .
\end{aligned}
$$

We first evaluate $\Sigma_{10}$. Since the $a, b$-sum is equal to 1 , by Lemma 2.1 we have

$$
\Sigma_{10} \ll \sum_{n \leq(q \tau)^{1+\epsilon}} \frac{(q \tau)^{\theta+\epsilon}}{n^{1-\sigma_{0}}} \sum_{m \leq(q \tau)^{\frac{1}{2}+\epsilon}} \frac{1}{m^{\sigma_{0}}} \ll(q \tau)^{\frac{1}{2}+\frac{\sigma_{0}}{2}+\theta+\epsilon} .
$$

Next, we evaluate $\Sigma_{9}$. 
I) First, we consider the case $N<(q \tau)^{\frac{15}{26}}, M<(q \tau)^{\frac{1}{4}}$. Since the $a$-sum is a Kloosterman sum, by Weil bound, its absolute value is at most $2 q^{\frac{1}{2}}$. Hence in this case we have

$$
\Sigma_{9} \ll q^{\frac{1}{2}} \sum_{n \ll N} \frac{N^{\theta+\epsilon}}{n^{1-\sigma_{0}}} \sum_{m \ll M} \frac{1}{m^{\sigma_{0}}} \ll q^{\frac{1}{2}} N^{\theta+\sigma_{0}+\epsilon} M^{1-\sigma_{0}} \ll q^{\frac{1}{2}}(q \tau)^{\frac{1}{4}+\frac{15}{26} \theta+\frac{17}{52} \sigma_{0}+\epsilon} .
$$

II) Next, we consider the case $M \geq(q \tau)^{\frac{1}{4}}$. We remove the condition $(m, q)=1$. Then the cost in the $m$-sum is at most

$$
\sum_{m^{\prime}=1}^{\infty} \frac{1}{\left(q m^{\prime}\right)^{\sigma_{0}}}\left|V_{\overline{s_{0}}}\left(\sqrt{q} m^{\prime}\right)\right| \mathcal{W}_{2}\left(\frac{q m^{\prime}}{M}\right) \ll q^{-\sigma_{0}} \sum_{m^{\prime} \ll \frac{M}{q}} \frac{1}{m^{\prime \sigma_{0}}} \ll q^{-1} M^{1-\sigma_{0}} .
$$

Hence

$$
\begin{aligned}
& \sum_{\substack{m \\
(m, q)=1}} \frac{1}{m^{\overline{s_{0}}}} V_{\overline{s_{0}}}\left(\frac{m}{\sqrt{q}}\right) \mathcal{W}_{2}\left(\frac{m}{M}\right) e\left(\frac{ \pm \bar{a} n m}{q}\right) \\
& =\sum_{m} \frac{1}{m^{\overline{s_{0}}}} V_{\overline{s_{0}}}\left(\frac{m}{\sqrt{q}}\right) \mathcal{W}_{2}\left(\frac{m}{M}\right) e\left(\frac{ \pm \bar{a} n m}{q}\right)+O\left(q^{-1} M^{1-\sigma_{0}}\right) .
\end{aligned}
$$

By Poisson summation, the $m$-sum becomes

$$
\begin{aligned}
& \sum_{m} \frac{1}{m^{\overline{s_{0}}}} V_{\overline{s_{0}}}\left(\frac{m}{\sqrt{q}}\right) \mathcal{W}_{2}\left(\frac{m}{M}\right) e\left(\frac{ \pm \bar{a} n m}{q}\right) \\
& =\sum_{b=1}^{q} e\left(\frac{ \pm \bar{a} b n}{q}\right) \sum_{m=-\infty}^{\infty} \frac{1}{(b+m q)^{\overline{s_{0}}}} V_{\overline{s_{0}}}\left(\frac{b+m q}{\sqrt{q}}\right) \mathcal{W}_{2}\left(\frac{b+m q}{M}\right) \\
& =\sum_{b=1}^{q} e\left(\frac{ \pm \bar{a} b n}{q}\right) \sum_{m=-\infty}^{\infty} \int_{-\infty}^{\infty}(b+q x)^{-\overline{s_{0}}} V_{\overline{s_{0}}}\left(\frac{b+q x}{\sqrt{q}}\right) \mathcal{W}_{2}\left(\frac{b+q x}{M}\right) e(-m x) d x
\end{aligned}
$$

Changing the variable by $(b+q x) / M=y$, (3.44) becomes

$$
\begin{aligned}
& \frac{M}{q} \sum_{m=-\infty}^{\infty} \sum_{b(\bmod q)} e\left(\frac{ \pm \bar{a} b n+m b}{q}\right) \\
& \cdot \int_{-\infty}^{\infty}(M y)^{-\overline{s_{0}}} V_{\overline{s_{0}}}\left(\frac{M y}{\sqrt{q}}\right) \mathcal{W}_{2}(y) e\left(-\frac{m M y}{q}\right) d y .
\end{aligned}
$$

Put

$$
I_{m}:=\int_{-\infty}^{\infty} y^{-\overline{s_{0}}} V_{\overline{s_{0}}}\left(\frac{M y}{\sqrt{q}}\right) \mathcal{W}_{2}(y) e\left(-\frac{m M y}{q}\right) d y
$$

If $m \neq 0$, by partial integration, we have

$$
\begin{aligned}
I_{m} & =(-1)^{B} \int_{-\infty}^{\infty} \frac{d^{B}}{d y^{B}}\left(y^{-\overline{s_{0}}} V_{\overline{s_{0}}}\left(\frac{M y}{\sqrt{q}}\right) \mathcal{W}_{2}(y)\right)\left(-\frac{q}{2 \pi i m M}\right)^{B} e\left(-\frac{m M y}{q}\right) d y \\
& \ll\left(\frac{q}{|m| M}\right)^{B} \int_{-\infty}^{\infty}\left|\frac{d^{B}}{d y^{B}}\left(y^{-\overline{s_{0}}} V_{\overline{s_{0}}}\left(\frac{M y}{\sqrt{q}}\right) \mathcal{W}_{2}(y)\right)\right| d y
\end{aligned}
$$


for any positive integer $B$. Moreover, for $y \in[1,2]$,

$$
\begin{aligned}
\frac{d^{B}}{d y^{B}}\left(y^{-\overline{s_{0}}} V_{\overline{s_{0}}}\left(\frac{M y}{\sqrt{q}}\right) \mathcal{W}_{2}(y)\right) & \ll\left(\frac{M}{\sqrt{q}}\right)^{B}\left|V_{\overline{s_{0}}}^{(B)}\left(\frac{M y}{\sqrt{q}}\right)\right|+\left|\frac{d^{B}}{d y^{B}}\left(y^{-\overline{s_{0}}}\right) V_{\overline{s_{0}}}\left(\frac{M y}{\sqrt{q}}\right)\right| \\
& \ll\left(\frac{M}{\sqrt{q}}\right)^{B}\left(\frac{M y}{\sqrt{q}}\right)^{-B}+\tau^{B} \ll \tau^{B} .
\end{aligned}
$$

Hence

$$
I_{m} \ll\left(\frac{q \tau}{|m| M}\right)^{B}
$$

for any $B>0$. Consequently, the contribution of the terms with $|m|>(q \tau)^{1+\epsilon} / M$ to $\Sigma_{9}$ is at most

$$
q \sum_{n} \frac{n^{\theta+\epsilon}}{n^{1-\sigma_{0}}}\left|W_{1-s_{0}}\left(\frac{n}{q}\right)\right| \cdot q \cdot \frac{M}{q} \cdot M^{-\sigma_{0}} \sum_{m>\frac{(q \tau) 1+\epsilon}{M}}\left(\frac{q \tau}{m M}\right)^{B} \ll(q \tau)^{-A} .
$$

Hence by (3.45), we have

$$
\begin{aligned}
\Sigma_{9}= & \sum_{\substack{n \geq 1 \\
(n, q)=1}} \frac{\lambda_{f}(n)}{n^{1-s_{0}}} W_{1-s_{0}}\left(\frac{n}{q}\right) \mathcal{W}_{1}\left(\frac{n}{N}\right) \sum_{a(\bmod q)}{ }^{*} e\left(\frac{a}{q}\right) \\
& \cdot\left\{\frac{M}{q} \sum_{|m| \leq \frac{(q \tau)}{M}} \sum_{b(\bmod q)} e\left(\frac{ \pm \bar{a} b n+m b}{q}\right)\right. \\
& \cdot \int_{-\infty}^{\infty}(M y)^{-\overline{s_{0}}} V_{\overline{s_{0}}}\left(\frac{M y}{\sqrt{q}}\right) \mathcal{W}_{2}(y) e\left(-\frac{m M y}{q}\right) d y \\
& \left.+O\left(q^{-1} M^{1-\sigma_{0}}\right)\right\}+O\left((q \tau)^{-A}\right) .
\end{aligned}
$$

The contribution of $O\left(q^{-1} M^{1-\sigma_{0}}\right)$ to $\Sigma_{9}$ is at most

$$
q \sum_{n \leq N} \frac{N^{\theta+\epsilon}}{n^{1-\sigma_{0}}} \cdot q^{-1} M^{1-\sigma_{0}} \ll N^{\sigma_{0}+\theta+\epsilon} M^{1-\sigma_{0}} .
$$

Moreover, the $b$-sum in (3.46) is equal to $q$ if $a \equiv \pm n \bar{m}(\bmod q)$ and otherwise 0 . Hence we have

$$
\begin{aligned}
\Sigma_{9}= & M^{1-\overline{s_{0}}} \sum_{\substack{n \geq 1 \\
(n, q)=1}} \frac{\lambda_{f}(n)}{n^{1-s_{0}}} W_{1-s_{0}}\left(\frac{n}{q}\right) \mathcal{W}_{1}\left(\frac{n}{N}\right) \\
& \cdot \sum_{|m| \leq \frac{(q \tau)}{M}} e\left( \pm \frac{n \bar{m}}{q}\right) \int_{-\infty}^{\infty} y^{-\overline{s_{0}}} V_{\overline{s_{0}}}\left(\frac{M y}{\sqrt{q}}\right) \mathcal{W}_{2}(y) e\left(-\frac{m M y}{q}\right) d y \\
& +O\left(N^{\sigma_{0}+\theta+\epsilon} M^{1-\sigma_{0}}\right) .
\end{aligned}
$$

We remove the condition $(n, q)=1$. Then the cost is at most

$$
M^{1-\sigma_{0}} \sum_{n^{\prime}=1}^{\infty} \frac{\left(q n^{\prime}\right)^{\theta+\epsilon}}{\left(q n^{\prime}\right)^{1-\sigma_{0}}}\left|W_{1-s_{0}}\left(n^{\prime}\right)\right| \cdot \frac{(q \tau)^{1+\epsilon}}{M} \ll q^{-1} M^{-\sigma_{0}}(q \tau)^{\sigma_{0}+\theta+1+\epsilon} .
$$


Combining this and (3.47), we have

$$
\begin{aligned}
\Sigma_{9} \ll & M^{1-\sigma_{0}} \sum_{|m| \leq \frac{(q \tau)}{M}}\left|\sum_{n \geq 1} \frac{\lambda_{f}(n)}{n^{1-s_{0}}} W_{1-s_{0}}\left(\frac{n}{q}\right) \mathcal{W}_{1}\left(\frac{n}{N}\right) e\left( \pm \frac{n \bar{m}}{q}\right)\right| \\
& +O\left(N^{\sigma_{0}+\theta+\epsilon} M^{1-\sigma_{0}}+q^{-1} M^{-\sigma_{0}}(q \tau)^{\sigma_{0}+\theta+1+\epsilon}\right) .
\end{aligned}
$$

By partial summation, the $n$-sum in (3.48) is

$$
\begin{aligned}
& \sum_{n \geq 1} \frac{\lambda_{f}(n)}{n^{1-s_{0}}} W_{1-s_{0}}\left(\frac{n}{q}\right) \mathcal{W}_{1}\left(\frac{n}{N}\right) e\left( \pm \frac{n \bar{m}}{q}\right) \\
& =\int_{N}^{2 N} u^{-1+s_{0}} W_{1-s_{0}}\left(\frac{u}{q}\right) \mathcal{W}_{1}\left(\frac{u}{N}\right) d\left(\sum_{n \leq u} \lambda_{f}(n) e\left( \pm \frac{n \bar{m}}{q}\right)\right) \\
& =-\int_{N}^{2 N}\left(\sum_{n \leq u} \lambda_{f}(n) e\left( \pm \frac{n \bar{m}}{q}\right)\right) \frac{d}{d u}\left(u^{-1+s_{0}} W_{1-s_{0}}\left(\frac{u}{q}\right) \mathcal{W}_{1}\left(\frac{u}{N}\right)\right) d u
\end{aligned}
$$

Combining Lemma 2.5 and the estimates $W_{1-s_{0}}(x) \ll 1, W_{1-s_{0}}^{\prime}(x) \ll x^{-1}$, we easily find that the above integral is $O\left(\tau(q \tau)^{\sigma_{0}-\frac{1}{2}+\epsilon}\right)$. Consequently by (3.48), we obtain

$$
\begin{aligned}
\Sigma_{9} & \ll M^{-\sigma_{0}} q^{-1}(q \tau)^{\sigma_{0}+\frac{3}{2}+\epsilon}+N^{\sigma_{0}+\theta+\epsilon} M^{1-\sigma_{0}} \\
& \ll q^{-1}(q \tau)^{\frac{3}{4} \sigma_{0}+\frac{3}{2}+\epsilon}+(q \tau)^{\frac{1}{2}+\frac{\sigma_{0}}{2}+\theta+\epsilon} .
\end{aligned}
$$

III) Finally, we consider the case $N \geq(q \tau)^{\frac{15}{26}}, M<(q \tau)^{\frac{1}{4}}$. In the evaluation of $\Sigma_{9}$, we remove the condition $(n, q)=1$. Then the cost is at most

$$
\begin{aligned}
& q \sum_{n^{\prime}} \sum_{m} \frac{\left(q n^{\prime}\right)^{\theta+\epsilon}}{\left(q n^{\prime}\right)^{1-\sigma_{0}}}\left|W_{1-s_{0}}\left(n^{\prime}\right)\right| \frac{1}{m^{\sigma_{0}}}\left|V_{\overline{s_{0}}}\left(\frac{m}{\sqrt{q}}\right)\right| \\
& \ll q^{\sigma_{0}+\theta+\epsilon} \sum_{n^{\prime} \leq \tau^{1+\epsilon}} n^{\prime-1+\sigma_{0}+\theta+\epsilon} \sum_{m \leq(q \tau)^{\frac{1}{2}+\epsilon}} m^{-\sigma_{0}} \ll(q \tau)^{\frac{1}{2}+\frac{\sigma_{0}}{2}+\theta+\epsilon .}
\end{aligned}
$$

Hence

$$
\begin{aligned}
\Sigma_{9}= & \sum_{\substack{m \\
(m, q)=1}} \frac{1}{m^{\overline{s_{0}}}} V_{\overline{s_{0}}}\left(\frac{m}{\sqrt{q}}\right) \mathcal{W}_{2}\left(\frac{m}{M}\right) \sum_{a(\bmod q)}^{*} e\left(\frac{a}{q}\right) \\
& \sum_{n} \frac{\lambda_{f}(n)}{n^{1-s_{0}}} W_{1-s_{0}}\left(\frac{n}{q}\right) \mathcal{W}_{1}\left(\frac{n}{N}\right) e\left( \pm \frac{\bar{a} n m}{q}\right)+O\left((q \tau)^{\frac{1}{2}+\frac{\sigma_{0}}{2}+\theta+\epsilon}\right) .
\end{aligned}
$$

To compute the $n$-sum above, we adapt Lemma 2.4 with $\psi(x)=x^{-1+s_{0}} W_{1-s_{0}}(N x / q) \mathcal{W}_{1}(x)$, $d= \pm a \bar{m}$. Then

$$
\begin{aligned}
& \sum_{n} \frac{\lambda_{f}(n)}{n^{1-s_{0}}} W_{1-s_{0}}\left(\frac{n}{q}\right) \mathcal{W}_{1}\left(\frac{n}{N}\right) e\left( \pm \frac{\bar{a} m n}{q}\right) \\
& =N^{-1+s_{0}}\left\{q \sum_{n=1}^{\infty} \frac{\lambda_{f}(n)}{n} e\left( \pm \frac{a \bar{m} n}{q}\right) \Psi_{+}\left(\frac{n N}{q^{2}}\right)\right. \\
& \left.+q \sum_{n=1}^{\infty} \frac{\lambda_{f}(n)}{n} e\left(\mp \frac{a \bar{m} n}{q}\right) \Psi_{-}\left(\frac{n N}{q^{2}}\right)\right\},
\end{aligned}
$$


where

$$
\Psi_{ \pm}(x)=\frac{1}{2 \pi i} \int_{(2)}\left(\pi^{2} x\right)^{-s} G_{ \pm}(s) \int_{0}^{\infty} t^{-1+s_{0}} W_{1-s_{0}}\left(\frac{N t}{q}\right) \mathcal{W}_{1}(t) t^{-s-1} d t d s
$$

We denote the contributions of the terms involving $\Psi_{+}, \Psi_{-}$to $\Sigma_{9}$ by $\Sigma_{9}^{+}, \Sigma_{9}^{-}$respectively and decompose

$$
\Sigma_{9}=\Sigma_{9}^{+}+\Sigma_{9}^{-}+O\left((q \tau)^{\frac{1}{2}+\frac{\sigma_{0}}{2}+\theta+\epsilon}\right) .
$$

We only evaluate $\Sigma_{9}^{+}$, since $\Sigma_{9}^{-}$is evaluated in the same way. Now $\Sigma_{9}^{+}$is given by

$$
\begin{aligned}
\Sigma_{9}^{+}= & q N^{-1+s_{0}} \sum_{\substack{m \\
(m, q)=1}} \frac{1}{m^{\overline{s_{0}}}} V_{\overline{s_{0}}}\left(\frac{m}{\sqrt{q}}\right) \mathcal{W}_{2}\left(\frac{m}{M}\right) \\
& \cdot \sum_{n=1}^{\infty} \frac{\lambda_{f}(n)}{n} \Psi_{+}\left(\frac{n N}{q^{2}}\right) \sum_{a(\bmod q)}^{*} e\left(\frac{a \pm a \bar{m} n}{q}\right) .
\end{aligned}
$$

The $a$-sum is equal to $q-1$ if $n \equiv \pm m(\bmod q)$ and otherwise -1 . Hence

$$
\begin{aligned}
\Sigma_{9}^{+}= & q(q-1) N^{-1+s_{0}} \sum_{\substack{n \\
(m, q)=1 \\
n \equiv \pm m(\bmod q)}} \frac{1}{m^{\overline{s 0}}} V_{\overline{s_{0}}}\left(\frac{m}{\sqrt{q}}\right) \mathcal{W}_{2}\left(\frac{m}{M}\right) \frac{\lambda_{f}(n)}{n} \Psi_{+}\left(\frac{n N}{q^{2}}\right) \\
& -q N^{-1+s_{0}} \sum_{\substack{n \\
(m, q)=1 \\
n \neq \pm m(\bmod q)}} \frac{1}{m^{\overline{s_{0}}}} V_{\overline{s_{0}}}\left(\frac{m}{\sqrt{q}}\right) \mathcal{W}_{2}\left(\frac{m}{M}\right) \frac{\lambda_{f}(n)}{n} \Psi_{+}\left(\frac{n N}{q^{2}}\right) \\
= & q^{2} N^{-1+s_{0}} \sum_{\substack{m \\
(m, q)=1}} \frac{1}{m^{\bar{s}}} V_{\overline{s_{0}}}\left(\frac{m}{\sqrt{q}}\right) \mathcal{W}_{2}\left(\frac{m}{M}\right) \sum_{n= \pm m(\bmod q)} \frac{\lambda_{f}(n)}{n} \Psi_{+}\left(\frac{n N}{q^{2}}\right) \\
& -q N^{-1+s_{0}} \sum_{\substack{m \\
(m, q)=1}} \frac{1}{m^{\overline{s_{0}}}} V_{\overline{s_{0}}}\left(\frac{m}{\sqrt{q}}\right) \mathcal{W}_{2}\left(\frac{m}{M}\right) \sum_{n} \frac{\lambda_{f}(n)}{n} \Psi_{+}\left(\frac{n N}{q^{2}}\right) \\
= & : q^{2} N^{-1+s_{0}} \sum_{9,1}^{+}-q N^{-1+s_{0}} \Sigma_{9,2}^{+},
\end{aligned}
$$

say. By partial integration, the $t$-integral in the definition of $\Psi_{+}(x)$ is evaluated by

$$
\int_{0}^{\infty} t^{-1+s_{0}} W_{1-s_{0}}\left(\frac{N t}{q}\right) \mathcal{W}_{1}(t) t^{-s-1} d t \ll \frac{1}{(|s|+1)^{B}}
$$

for any $B>0$. In addition, by Stirling's estimate we have

$$
G_{+}(s) \ll(|s|+1)^{2 \Re(s)+1} .
$$

Hence by moving the path of integration in $s$ in (3.52) to $\Re(s)=C(0<C<B / 3)$, we find that

$$
\Psi_{+}(x) \ll \int_{(C)}\left|\left(\pi^{2} x\right)^{-s}(1+|s|)^{2 C+1}\right|(1+|s|)^{-B}|d s| \ll x^{-C} .
$$

Therefore, the contributions of the terms with $n \geq(q \tau)^{2+\epsilon} / N$ to $\Sigma_{9,1}^{+}, \Sigma_{9,2}^{+}$are at most $O\left((q \tau)^{-A}\right)$. On the other hand, by moving the path of integration in $s$ in (3.52) 
to $\Re(s)=-1+\epsilon$, we have $\Psi_{+}(x) \ll q^{\epsilon} x$. Hence

$$
\begin{aligned}
\Sigma_{9,1}^{+} & \ll \sum_{M \leq m \leq 2 M} \frac{1}{m^{\sigma_{0}}} \sum_{\substack{n \leq \frac{(q \tau)^{2+\epsilon}}{N} \\
n \equiv \pm m(\bmod q)}} \frac{\left|\lambda_{f}(n)\right|}{n} q^{\epsilon} \frac{n N}{q^{2}} \ll M^{1-\sigma_{0}} q^{-2+\epsilon} N \sup _{m} \sum_{\substack{n \leq \frac{(q \tau)^{2+\epsilon}}{N} \\
n \equiv \pm m(\bmod q)}} n^{\theta+\epsilon} \\
& \ll M^{1-\sigma_{0}} N^{-\theta} q^{-3}(q \tau)^{2 \theta+2+\epsilon}, \\
\Sigma_{9,2}^{+} & \ll \sum_{M \leq m \leq 2 M} \frac{1}{m^{\sigma_{0}}} \sum_{n \leq \frac{(q \tau)^{2+\epsilon}}{N}} \frac{\left|\lambda_{f}(n)\right|}{n} q^{\epsilon} \frac{n N}{q^{2}} \ll M^{1-\sigma_{0}} N q^{-2+\epsilon} \sum_{n \leq \frac{(q \tau)^{2+\epsilon}}{N}} n^{\theta+\epsilon} \\
& \ll M^{1-\sigma_{0}} N^{-\theta} q^{-2}(q \tau)^{2+2 \theta+\epsilon} .
\end{aligned}
$$

Therefore, by (3.55) we have

$$
\Sigma_{9}^{+} \ll q^{-1} M^{1-\sigma_{0}} N^{\sigma_{0}-\theta-1}(q \tau)^{2 \theta+2+\epsilon} .
$$

As we mentioned before, the same estimate holds for $\Sigma_{9}^{-}$. Hence by (3.53) and our assumption $M<(q \tau)^{\frac{1}{4}}, N \geq(q \tau)^{\frac{15}{26}}$, we have

$$
\begin{aligned}
\Sigma_{9} & \ll q^{-1} M^{1-\sigma_{0}} N^{\sigma_{0}-\theta-1}(q \tau)^{2 \theta+2+\epsilon}+(q \tau)^{\frac{1}{2}+\frac{\sigma_{0}}{2}+\theta+\epsilon} \\
& \ll q^{-1}(q \tau)^{\frac{17}{52} \sigma_{0}+\frac{37}{26} \theta+\frac{87}{52}+\epsilon}+(q \tau)^{\frac{1}{2}+\frac{\sigma_{0}}{2}+\theta+\epsilon} .
\end{aligned}
$$

Combining (3.42), (3.49), (3.56), we obtain

$$
\begin{aligned}
q^{-2 s_{0}}(q-1) \Sigma_{9} \ll & q^{\frac{3}{2}-2 \sigma_{0}}(q \tau)^{\frac{1}{4}+\frac{15}{26} \theta+\frac{17}{52} \sigma_{0}+\epsilon}+q^{-2 \sigma_{0}}(q \tau)^{\frac{3}{4} \sigma_{0}+\frac{3}{2}+\epsilon} \\
& +q^{1-2 \sigma_{0}}(q \tau)^{\frac{1}{2}+\frac{\sigma_{0}}{2}+\theta+\epsilon}+q^{-2 \sigma_{0}}(q \tau)^{\frac{17}{52} \sigma_{0}+\frac{37}{26} \theta+\frac{87}{52}+\epsilon}
\end{aligned}
$$

Substituting (3.41), (3.57) into (3.40) and combining the estimate of the quotient of gamma factors, we conclude that the left hand side of (3.38) is

$$
\begin{aligned}
& \sum_{\chi(\bmod q)}^{*}(\chi(-1)+1) S_{2} S_{3} \\
& \ll \tau^{1-2 \sigma_{0}}\left\{q^{-2 \sigma_{0}}(q \tau)^{\frac{1}{2}+\frac{\sigma_{0}}{2}+\theta+\epsilon}+q^{\frac{3}{2}-2 \sigma_{0}}(q \tau)^{\frac{1}{4}+\frac{15}{26} \theta+\frac{17}{52} \sigma_{0}+\epsilon}\right. \\
& \left.\quad+q^{-2 \sigma_{0}}(q \tau)^{\frac{3}{4} \sigma_{0}+\frac{3}{2}+\epsilon}+q^{1-2 \sigma_{0}}(q \tau)^{\frac{1}{2}+\frac{\sigma_{0}}{2}+\theta+\epsilon}+q^{-2 \sigma_{0}}(q \tau)^{\frac{17}{52} \sigma_{0}+\frac{37}{26} \theta+\frac{87}{52}+\epsilon}\right\} \\
& \ll q^{-1}(q \tau)^{\frac{3}{2}-\frac{3}{2} \sigma_{0}+\theta+\epsilon}+q^{\frac{1}{2}}(q \tau)^{\frac{5}{4}+\frac{15}{26} \theta-\frac{87}{52} \sigma_{0}+\epsilon}+q^{-1}(q \tau)^{\frac{5}{2}-\frac{5}{4} \sigma_{0}+\epsilon} \\
& \quad+(q \tau)^{\frac{3}{2}-\frac{3}{2} \sigma_{0}+\theta+\epsilon}+q^{-1}(q \tau)^{-\frac{87}{52} \sigma_{0}+\frac{37}{26} \theta+\frac{139}{52}+\epsilon} .
\end{aligned}
$$

Since the first and fourth terms of the right hand side of (3.58) are dominated by the third, we obtain (3.37).

By substituting (3.21), (3.22), (3.30) and (3.37) into (3.1), we obtain (1.2).

\section{The proof of Theorem $\mathbf{1 . 3}$}

To prove Theorem 1.3, we need some upper bounds for the moments of $L$ functions at $s=s_{0}$, and to prove these estimates we use the following well known inequality. 
Lemma 4.1. For any complex numbers $a_{n}(n=1,2,3, \cdots)$, we have

$$
\sum_{\chi(\bmod q)}\left|\sum_{n \leq N} a_{n} \chi(n)\right|^{2} \leq(q+N) \sum_{n \leq N}\left|a_{n}\right|^{2} .
$$

Applying the above lemma to the approximate functional equation of $L\left(s_{0}, f \otimes \chi\right)$, we obtain the following estimate.

Proposition 4.2. Let $f$ be an even Hecke-Maass form of $\operatorname{SL}(2, \mathbf{Z})$. Then, for $s_{0}=\sigma_{0}+i t_{0}$, we have

$$
\sum_{\chi(\bmod q)}^{+}\left|L\left(s_{0}, f \otimes \chi\right)\right|^{2} \ll \begin{cases}(q \tau)^{1+\epsilon} & \left(\sigma_{0}=\frac{1}{2}\right), \\ q \tau & \left(\frac{1}{2}<\sigma_{0}<1\right) .\end{cases}
$$

Proof. By Lemma 2.7, we have

$$
\begin{aligned}
& L\left(s_{0}, f \otimes \chi\right) \\
& =\mathcal{L}_{1}\left(s_{0}, \chi\right)+\mathcal{L}_{2}\left(s_{0}, \chi\right)+\tau(\chi)^{2} q^{-2 s_{0}} \frac{\tilde{\gamma}\left(1-s_{0}\right)}{\tilde{\gamma}\left(s_{0}\right)}\left(\mathcal{L}_{3}\left(s_{0}, \chi\right)+\mathcal{L}_{4}\left(s_{0}, \chi\right)\right)+O\left((q \tau)^{-A}\right),
\end{aligned}
$$

where

$$
\begin{aligned}
& \mathcal{L}_{1}\left(s_{0}, \chi\right)=\sum_{n \leq q \tau} \frac{\lambda_{f}(n) \chi(n)}{n^{s_{0}}} W_{s_{0}}\left(\frac{n}{q}\right), \\
& \mathcal{L}_{2}\left(s_{0}, \chi\right)=\sum_{q \tau<n \leq(q \tau)^{1+\epsilon}} \frac{\lambda_{f}(n) \chi(n)}{n^{s_{0}}} W_{s_{0}}\left(\frac{n}{q}\right), \\
& \mathcal{L}_{3}\left(s_{0}, \chi\right)=\sum_{n \leq q \tau} \frac{\lambda_{f}(n) \bar{\chi}(n)}{n^{1-s_{0}}} W_{1-s_{0}}\left(\frac{n}{q}\right), \\
& \mathcal{L}_{4}\left(s_{0}, \chi\right)=\sum_{q \tau<n \leq(q \tau)^{1+\epsilon}} \frac{\lambda_{f}(n) \bar{\chi}(n)}{n^{1-s_{0}}} W_{1-s_{0}}\left(\frac{n}{q}\right) .
\end{aligned}
$$

Hence

$$
\begin{aligned}
\sum_{\chi(\bmod q)}^{+}\left|L\left(s_{0}, f \otimes \chi\right)\right|^{2} \ll & \sum_{\chi(\bmod q)}\left|\mathcal{L}_{1}\left(s_{0}, \chi\right)\right|^{2}+\sum_{\chi(\bmod q)}\left|\mathcal{L}_{2}\left(s_{0}, \chi\right)\right|^{2} \\
+ & |\tau(\chi)|^{4} q^{-4 \sigma_{0}}\left|\frac{\tilde{\gamma}\left(1-s_{0}\right)}{\tilde{\gamma}\left(s_{0}\right)}\right|^{2} \\
& \cdot\left(\sum_{\chi(\bmod q)}\left|\mathcal{L}_{3}\left(s_{0}, \chi\right)\right|^{2}+\sum_{\chi(\bmod q)}\left|\mathcal{L}_{4}\left(s_{0}, \chi\right)\right|^{2}\right) .
\end{aligned}
$$

We evaluate the above four moments of $L$-functions by combining Lemma 4.1 and the Rankin-Selberg type estimate

$$
\sum_{n \leq x}\left|\lambda_{f}(n)\right|^{2} \ll x
$$


First,

$$
\begin{aligned}
& \sum_{\chi(\bmod q)}\left|\mathcal{L}_{1}\left(s_{0}, \chi\right)\right|^{2}=\sum_{\chi(\bmod q)}\left|\sum_{n \leq q \tau} \frac{\lambda_{f}(n) \chi(n)}{n^{s_{0}}} W_{s_{0}}\left(\frac{n}{q}\right)\right|^{2} \\
& \leq(q+q \tau) \sum_{n \leq q \tau} \frac{\left|\lambda_{f}(n)\right|^{2}}{n^{2 \sigma_{0}}}=(q+q \tau) \int_{1-}^{q \tau} u^{-2 \sigma_{0}} d\left(\sum_{n \leq u}\left|\lambda_{f}(n)\right|^{2}\right) \\
& =(q+q \tau)\left\{\left[u^{-2 \sigma_{0}} \sum_{n \leq u}\left|\lambda_{f}(n)\right|^{2}\right]_{1-}^{q \tau}+2 \sigma_{0} \int_{1-}^{q \tau} u^{-2 \sigma_{0}-1} \sum_{n \leq u}\left|\lambda_{f}(n)\right|^{2} d u\right\} \\
& \ll q \tau \int_{1}^{q \tau} u^{-2 \sigma_{0}} d u \ll \begin{cases}q \tau \log q \tau & \left(\sigma_{0}=\frac{1}{2}\right), \\
q \tau & \left(\sigma_{0}>\frac{1}{2}\right) .\end{cases}
\end{aligned}
$$

Next,

$$
\begin{aligned}
\sum_{\chi(\bmod q)}\left|\mathcal{L}_{2}\left(s_{0}, \chi\right)\right|^{2}= & \sum_{\chi(\bmod q)}\left|\sum_{q \tau<n \leq(q \tau)^{1+\epsilon}} \frac{\lambda_{f}(n) \chi(n)}{n^{s_{0}}} W_{s_{0}}\left(\frac{n}{q}\right)\right|^{2} \\
\ll & \ll(q \tau)^{1+\epsilon} \sum_{q \tau<n \leq(q \tau)^{1+\epsilon}} \frac{\left|\lambda_{f}(n)\right|^{2}}{n^{2 \sigma_{0}}}\left(\frac{n}{q \tau}\right)^{-A} \\
= & (q \tau)^{A+1+\epsilon} \int_{q \tau}^{(q \tau)^{1+\epsilon}} u^{-2 \sigma_{0}-A} d\left(\sum_{q \tau<n \leq u}\left|\lambda_{f}(n)\right|^{2}\right) \\
= & (q \tau)^{A+1+\epsilon}\left\{\left[u^{-2 \sigma_{0}-A} \sum_{q \tau<n \leq u}\left|\lambda_{f}(n)\right|^{2}\right]_{q \tau}^{(q \tau)^{1+\epsilon}}\right. \\
& \left.+\left(2 \sigma_{0}+A\right) \int_{q \tau}^{(q \tau)^{1+\epsilon}} u^{-2 \sigma_{0}-A-1} \sum_{q \tau<n \leq u}\left|\lambda_{f}(n)\right|^{2} d u\right\} \\
& \ll(q \tau)^{A+1+\epsilon} \int_{q \tau}^{(q \tau)^{1+\epsilon}} u^{-2 \sigma_{0}-A} d u \ll(q \tau)^{2-2 \sigma_{0}+\epsilon} .
\end{aligned}
$$

Next, since $\mathcal{L}_{3}\left(s_{0}, \chi\right)=\mathcal{L}_{1}\left(1-s_{0}, \bar{\chi}\right)$, by the evaluation of the moment of $\mathcal{L}_{1}\left(s_{0}, \chi\right)$ above, we have

$$
\sum_{\chi(\bmod q)}\left|\mathcal{L}_{3}\left(s_{0}, \chi\right)\right|^{2} \ll q \tau \int_{1}^{q \tau} u^{-2\left(1-\sigma_{0}\right)} d u \ll \begin{cases}q \tau \log q \tau & \left(\sigma_{0}=\frac{1}{2}\right), \\ (q \tau)^{2 \sigma_{0}} & \left(\sigma_{0}>\frac{1}{2}\right) .\end{cases}
$$

Finally, since $\mathcal{L}_{4}\left(s_{0}, \chi\right)=\mathcal{L}_{2}\left(1-s_{0}, \bar{\chi}\right)$, by the estimate of the moment of $\mathcal{L}_{2}\left(s_{0}, \chi\right)$ above, we have

$$
\sum_{\chi(\bmod q)}\left|\mathcal{L}_{4}\left(s_{0}, \chi\right)\right|^{2} \ll(q \tau)^{2 \sigma_{0}+\epsilon}
$$

Substituting these estimates and $|\tau(\chi)|=q^{\frac{1}{2}}, \tilde{\gamma}\left(1-s_{0}\right) / \tilde{\gamma}\left(s_{0}\right) \ll \tau^{1-2 \sigma_{0}}$ into (4.2), we obtain (4.1). 
Proposition 4.3. We have

$$
\sum_{\chi(\bmod q)}^{+}\left|L\left(s_{0}, \chi\right)\right|^{4} \ll \begin{cases}(q \tau)^{1+\epsilon} & \left(\sigma_{0}=\frac{1}{2}\right), \\ q \tau & \left(\frac{1}{2}<\sigma_{0}<1\right) .\end{cases}
$$

Proof. Let $\chi$ be a primitive Dirichlet character modulo $q$. Then $L\left(s_{0}, \chi\right)^{2}$ is expressed by

$$
L\left(s_{0}, \chi\right)^{2}=\sum_{n} \frac{\chi(n) d(n)}{n^{s_{0}}} U_{s_{0}}\left(\frac{n}{q}\right)+\tau(\chi)^{2} q^{-2 s_{0}} \frac{\gamma\left(1-s_{0}\right)^{2}}{\gamma\left(s_{0}\right)^{2}} \sum_{n} \frac{\bar{\chi}(n) d(n)}{n^{1-s_{0}}} U_{1-s_{0}}\left(\frac{n}{q}\right),
$$

where $\gamma(s)=\pi^{-\frac{s}{2}} \Gamma\left(\frac{s}{2}\right), d(n)$ denotes the number of positive divisors of $n$, and

$$
U_{s_{0}}(x)=\frac{1}{2 \pi i} \int_{(3)}(\pi x)^{-u} \frac{\Gamma\left(\frac{s_{0}+u}{2}\right)^{2}}{\Gamma\left(\frac{s_{0}}{2}\right)^{2}} e^{u^{2}} \frac{d u}{u} .
$$

The function $U_{s_{0}}(x)$ has the estimate

$$
U_{s_{0}}(x) \ll_{A} \min \left\{1,\left(\frac{x}{\tau}\right)^{-A}\right\}
$$

for any $A>0$. Hence we have

$$
\begin{aligned}
L\left(s_{0}, \chi\right)^{2}= & \mathcal{L}_{5}\left(s_{0}, \chi\right)+\mathcal{L}_{6}\left(s_{0}, \chi\right)+\tau(\chi)^{2} q^{-2 s_{0}} \frac{\gamma\left(1-s_{0}\right)^{2}}{\gamma\left(s_{0}\right)^{2}}\left(\mathcal{L}_{7}\left(s_{0}, \chi\right)\right. \\
& \left.+\mathcal{L}_{8}\left(s_{0}, \chi\right)\right)+O\left((q \tau)^{-A}\right)
\end{aligned}
$$

where

$$
\begin{aligned}
& \mathcal{L}_{5}\left(s_{0}, \chi\right)=\sum_{n \leq q \tau} \frac{\chi(n) d(n)}{n^{s_{0}}} U_{s_{0}}\left(\frac{n}{q}\right) \\
& \mathcal{L}_{6}\left(s_{0}, \chi\right)=\sum_{q \tau<n \leq(q \tau)^{1+\epsilon}} \frac{\chi(n) d(n)}{n^{s_{0}}} U_{s_{0}}\left(\frac{n}{q}\right) \\
& \mathcal{L}_{7}\left(s_{0}, \chi\right)=\sum_{n \leq q \tau} \frac{\bar{\chi}(n) d(n)}{n^{1-s_{0}}} U_{1-s_{0}}\left(\frac{n}{q}\right) \\
& \mathcal{L}_{8}\left(s_{0}, \chi\right)=\sum_{q \tau<n \leq(q \tau)^{1+\epsilon}} \frac{\bar{\chi}(n) d(n)}{n^{1-s_{0}}} U_{1-s_{0}}\left(\frac{n}{q}\right) .
\end{aligned}
$$

Consequently,

$$
\begin{aligned}
\sum_{\chi(\bmod q)}^{+}\left|L\left(s_{0}, \chi\right)\right|^{4} \ll & \sum_{\chi(\bmod q)}\left|\mathcal{L}_{5}\left(s_{0}, \chi\right)\right|^{2}+\sum_{\chi(\bmod q)}\left|\mathcal{L}_{6}\left(s_{0}, \chi\right)\right|^{2} \\
& +|\tau(\chi)|^{4} q^{-4 \sigma_{0}}\left|\frac{\gamma\left(1-s_{0}\right)}{\gamma\left(s_{0}\right)}\right|^{4} \\
& \cdot\left(\sum_{\chi(\bmod q)}\left|\mathcal{L}_{7}\left(s_{0}, \chi\right)\right|^{2}+\sum_{\chi(\bmod q)}\left|\mathcal{L}_{8}\left(s_{0}, \chi\right)\right|^{2}\right) .
\end{aligned}
$$


The methods to evaluate the moments of $\mathcal{L}_{5}, \cdots, \mathcal{L}_{8}$ are almost the same as those in the proof of Proposition 4.2 above. The only difference is that we use the estimate

$$
\sum_{n \leq x} d(n)^{2} \ll x(\log x)^{3}
$$

instead of (4.3). Thus we have

$$
\begin{aligned}
& \sum_{\chi(\bmod q)}\left|\mathcal{L}_{5}\left(s_{0}, \chi\right)\right|^{2} \ll \begin{cases}q \tau(\log q \tau)^{4} & \left(\sigma_{0}=\frac{1}{2}\right), \\
q \tau & \left(\frac{1}{2}<\sigma_{0}<1\right),\end{cases} \\
& \sum_{\chi(\bmod q)}\left|\mathcal{L}_{6}\left(s_{0}, \chi\right)\right|^{2} \ll(q \tau)^{2-2 \sigma_{0}+\epsilon}, \\
& \sum_{\chi(\bmod q)}\left|\mathcal{L}_{7}\left(s_{0}, \chi\right)\right|^{2} \ll \begin{cases}q \tau(\log q \tau)^{4} & \left(\sigma_{0}=\frac{1}{2}\right), \\
(q \tau)^{2 \sigma_{0}+\epsilon} & \left(\frac{1}{2}<\sigma_{0}<1\right),\end{cases} \\
& \sum_{\chi(\bmod q)}\left|\mathcal{L}_{8}\left(s_{0}, \chi\right)\right|^{2} \ll(q \tau)^{2 \sigma_{0}+\epsilon} .
\end{aligned}
$$

Substituting these estimates and $|\tau(\chi)|=q^{\frac{1}{2}}, \gamma\left(1-s_{0}\right) / \gamma\left(s_{0}\right) \ll \tau^{\frac{1}{2}-\sigma_{0}}$ into (4.5), we obtain (4.4).

We prove Theorem 1.3. Using the generalized Hölder's inequality, we have

$$
\begin{aligned}
& \left|\sum_{\chi(\bmod q)}^{+} L\left(s_{0}, f \otimes \chi\right) \overline{L\left(s_{0}, \chi\right)}\right| \\
& \leq\left|\mathcal{A}_{f, s_{0}}\right|^{\frac{1}{4}}\left(\sum_{\chi(\bmod q)}^{+}\left|L\left(s_{0}, f \otimes \chi\right)\right|^{2}\right)^{\frac{1}{2}}\left(\sum_{\chi(\bmod q)}^{+}\left|L\left(s_{0}, \chi\right)\right|^{4}\right)^{\frac{1}{4}} .
\end{aligned}
$$

If $q \gg \tau^{M\left(\sigma_{0}, \theta\right)+\epsilon}$, the left hand side of (4.6) is asymptotically $\asymp q$. By adapting Propositions 4.2-4.3, we obtain the conclusion of Theorem 1.3.

Acknowledgements. This work is partially supported by the JSPS, KAKENHI Grant Number 16K17574. The author sincerely expresses his gratitude to the referee for reading the first manuscript carefully and giving valuable comments and suggestions.

\section{References}

[1] Akbary, A.: Simultaneous non-vanishing of twists. - Proc. Amer. Math. Soc. 134:11, 2006, 3143-3151.

[2] BARThel, L., and D. RAmakrishnan: A nonvanishing result for twists of $L$-functions of GL(n). - Duke Math. J. 74, 1994, 681-700.

[3] Blomer, V., E. Fouvry, E. Kowalski, P. Michel, and D. Milićević: On moments of twisted $L$-functions. - Amer. J. Math. 139:3, 2017, 707-768.

[4] Das, S., and R. KHAN: Simultaneous nonvanishing of Dirichlet $L$-functions and twists of Hecke-Maass L-functions. - J. Ramanujan Math. Soc. 30:3, 2015, 237-250.

[5] Davenport, H.: Multiplicative number theory. Third edition. - Springer, New York, 2000.

[6] Godber, D.: Additive twists of Fourier coefficients of modular forms. - J. Number Theory 133:1, 2013, 83-104. 
[7] Hoffstein, J., and M. LeE: Second moments and simultaneous non-vanishing of GL(2) automorphic $L$-series. - Preprint, arXiv:1308.5980.

[8] Iwaniec, H.: Spectral methods of automorphic forms. Second edition. - Grad. Stud. Math. 53, Amer. Math. Soc., Providence, RI, 2002.

[9] Iwaniec, H., and E. Kowalski: Analytic number theory. - Amer. Math. Colloq. Publ. 53, Amer. Math. Soc., Providence, RI, 2004.

[10] Iwaniec, H., and P. SARnak: Dirichlet $L$-functions at the central point. - Number Theory in Progress 2,1997, 941-952.

[11] IwANiEc, H., and P. SARnAK: The non-vanishing of central values of automorphic $L$-functions and Landau-Siegel zeros. - Israel J. Math. 120:A, 2000, 155-177.

[12] Khan, R.: Simultaneous non-vanishing of GL(3) $\times$ GL(2) and GL(2) $L$-functions. - Math. Proc. Cambridge Philos. Soc. 152:3, 2012, 535-553.

[13] KIM, H. K.: Functoriality for the exteriror square of $\mathrm{GL}_{4}$ and the symmetric fourth of $\mathrm{GL}_{2}$. J. Amer. Math. Soc. 16:1, 2003, 139-183.

[14] LI, X.: The central value of Rankin-Selberg L-functions. - Geom. Funct. Anal. 18:5, 2009, 1660-1695.

[15] Liu, S.-C.: Simultaneous nonvanishing of GL(2) $\times$ GL(2) and GL(2) $L$-functions. - Proc. Amer. Math. Soc. 142, 2014, 1953-1964.

[16] LiU, S.-C.: Simultaneous nonvanishing of automorphic $L$-functions. - J. Number Theory 147, $2015,620-632$.

[17] Michel, P., and J. Vanderkam: Simultaneous nonvanishing of twists of automorphic $L$ functions. - Compos. Math. 134:2, 2002, 135-191.

[18] Munshi, R.: A note on simultaneous nonvanishing twists. - J. Number Theory 132, 2012, $666-674$.

[19] Ramakrishnan, D., and J. Rogawski: Average values of modular $L$-series via the relative trace formula. - Pure Appl. Math. Q. 1:4, 2005, 701-735.

[20] Rohrlish, D.: Nonvanishing of $L$-functions for GL(2). - Invent. Math. 97, 1989, 381-403.

[21] Soundararajan, K.: Nonvanishing of quadratic Dirichlet $L$-functions at $s=1 / 2$. - Ann. of Math. (2) 152:2, 2000, 447-488.

[22] Sun, Q.: A note on simultaneous nonvanishing of Dirichlet $L$-functions and twists of HeckeMaass L-functions. - Preprint, arXiv:1609.03091.

[23] Xu, Z.: Simultaneous nonvanishing of automorphic $L$-functions at the central point. Manuscripta Math. 134:3-4, 2011, 309-342. 